\title{
FINITE-DIMENSIONAL SOLITON SYSTEMS
}

\author{
S.N.M. Ruijsenaars \\ Centre for Mathematics and Computer Science \\ P.O. Box 4079, 1009 AB Amsterdam, The Netherlands
}

ABSTRACT. We survey recent results concerning Calogero-Moser and Toda systems and integrable generalizations thereof. We also discuss relations to infinite-dimensional integrable systems.

1. Introduction

2. Classical soliton systems

2A. Classical integrability and the soliton property

2B. Pure soliton systems

2B1. The Hamiltonian $H$

2B2. The Hamiltonians $S_{1}, \ldots, S_{N}$

2B3. The action-angle transform and duality

2B4. Soliton scattering

2C. Systems related to pure soliton systems

$2 \mathrm{Cl}$. Systems with solitons and antisolitons

$2 \mathrm{C} 2$. Sutherland type systems and their duals

2C3. Elliptic systems

2C4. Periodic Toda type systems

3. Quantum soliton systems

3A. Quantum integrability and the soliton property

3B. Quantized pure soliton systems

3B1. Preserving commutativity

3B2. The eigenfunction transform and duality

3B3. Soliton scattering

3C. Systems related to quantized pure soliton systems

$3 \mathrm{Cl}$. Systems with solitons and antisolitons

$3 \mathrm{C} 2$. Sutherland type systems and their duals

3C3. Elliptic systems

3C4. Periodic Toda type systems

4. Connections with infinite-dimensional integrable systems
4A. Preamble
4B. The classical level
4 C. The quantum level

Acknowledgements

References

- Work supported by the Netherlands Organisation for the Advancement of Research (NWO). 


\section{INTRODUCTION}

In recent years the well-known nonrelativistic Calogero-Moser and Toda $N$-particle systems have been shown to admit integrable relativistic generalizations [1-3]. Results on the former systems and their versions for root systems other than $A_{N-1}$ have been surveyed by Olshanetsky and Perelomov in the early eighties $[4,5]$. Here, we concentrate on results obtained since that time, especially as concerns the relativistic systems.

We presuppose no previous knowledge concerning finite-dimensional integrable systems, but some acquaintance with the surveys $[4,5]$ would probably be helpful. Also, we limit ourselves to the physically most relevant case of translation-invariant interactions. Thus, root systems other than $A_{N-1}$ are only peripherally mentioned, and no external field couplings preserving integrability are considered. Furthermore, internal degrees of freedom are not discussed and we do not deal with the thermodynamics associated with the systems.

Chapters 2 and 3 are concerned with the classical and the quantum versions, resp., of the class of integrable systems just delineated. In both chapters integrability issues and relations between the various systems are discussed in some detail. In Chapter 2 we also sketch our results on explicit actionangle transformations [6-8], which lead in particular to duality relations between various parameter regimes. These classical duality properties are of interest not only in their own right, but also because they have obvious quantum translations.

This is explained in Chapter 3, in connection with our description of explicit knowledge concerning the joint eigenfunction transform. This transform is the quantum analog of the action-angle transform, and its duality properties agree with those of the action-angle transform in all cases where this has been checked. In fact, our expectation that the classical self-duality of the "master parameter regime' at the 'one-period' level (the $\mathrm{II}_{\mathrm{red}}$ regime described below) will survive quantization has been a crucial guide towards finding explicit eigenfunctions, some of which will be reported here for the first time. As it turns out, the quest for a unitary eigenfunction transform for the relativistic systems leads into uncharted territory at the intersection of Hilbert space theory and the theory of analytic difference equations. Here, too, duality properties have been of considerable help.

In Chapters 2 and 3 we emphasize explicit knowledge concerning the action-angle and joint eigenfunction transforms, not only because we feel that these maps are the central mathematical objects in the systems at issue, but also because this knowledge is indispensable in making contact with the world of infinite-dimensional integrable systems. Chapter 4 is devoted to a sketch of some of the connections that have emerged thus far. We believe there is a lot more in store here, especially at the quantum level.

\section{CLASSICAL SOLTTON SYSTEMS}

2A. CLASSICAL INTEGRABLITY AND THE SOLTrON PROPERTY

To provide some context for our definition of 'soliton system' it is expedient to begin with some remarks on the more general notion of 'Liouville integrable system'. We shall restrict ourselves to the simplest type of phase space $\langle\Omega, \omega\rangle$, viz., the cotangent bundle over a region $G \subset \mathbf{R}^{N}$,

$$
\Omega \equiv\left\{(q, \theta) \in \mathbb{R}^{2 N} \mid q \in G\right\}
$$

with its obvious symplectic form

$$
\omega \equiv \sum_{j=1}^{N} d q_{j} \wedge d \theta_{j}
$$

Then a Hamiltonian $H$ on $\Omega$ defines a Liorville integrable system whenever there exist $N$ independent functions $S_{1}, \ldots, S_{N}$ on $\Omega$ such that

$$
\begin{aligned}
& \left\{H, S_{j}\right\}=0, \quad j=1, \ldots, N \\
& \left\{S_{j}, S_{k}\right\}=0, \quad j, k=1, \ldots, N .
\end{aligned}
$$


It is important to note that in the very general, mathematical context of this definition the construction of integrable systems is just as easy (or hard) as the construction of canonical transformations. Indeed, let $\Omega$ be a region in $\mathbb{R}^{2 N}$ with coordinates $(\hat{q}, \hat{\theta})$ and symplectic form

$$
\hat{\omega} \equiv \sum_{j=1}^{N} d \hat{q}_{j} \wedge d \hat{\theta}_{j} .
$$

Now let $\tilde{E}$ be a canonical map from $\hat{\Omega}$ onto $\Omega$, and define

$$
\begin{aligned}
& \hat{H}(\hat{q}, \hat{\theta}) \equiv f(\hat{\theta}) \\
& \hat{S}_{k}(\hat{q}, \hat{\theta}) \equiv \sum_{1<i_{1}<\cdots<i_{k}<N} \hat{\theta}_{i_{1}} \cdots \hat{\theta}_{i_{k}}, \quad k=1, \ldots, N
\end{aligned}
$$

where $f \in C_{\mathbb{R}}^{\infty}(\mathbb{R})$. Then the pullback Hamiltonians

$$
\begin{aligned}
& H \equiv \hat{H} \circ \Phi \\
& S_{k} \equiv \hat{S}_{k} \circ \Phi
\end{aligned}
$$

where

$$
\Phi \equiv \mathcal{E}^{-1},
$$

yield an integrable dynamics $H$ on $\Omega$ with commuting integrals $S_{1}, \ldots, S_{N}$. Conversely, the Liouville-Arnold theorem [9] implies that any integrable system $H_{1}, S_{1}, \ldots, S_{N}$ may be viewed as such a pullback. (Of course, the structure of $\langle\hat{\Omega}, \hat{\omega}\rangle$ will be far more complicated than just assumed, in general.)

The upshot is, that the class of Liouville integrable systems is in essence equivalent to the class of canonical transformations. (The qualifier cannot be omitted due to topological niceties we have no occasion to recall here.) We shall now head for a much stronger notion of integrability, which is however tied to a quite special, physical context.

To this end, we consider a Hamiltonian $H(q, \theta)$ that models the dynamics of $N$ interacting particles on the line. We assume that the interaction has a repulsive character. More precisely, we assume that the wave transformations $[10,11]$

$$
\varepsilon_{ \pm}:\left\langle\Omega^{ \pm}, \omega^{ \pm}>\rightarrow<\Omega, \omega>,\left(q^{ \pm}, \theta^{ \pm}\right) \mapsto(q, \theta)\right.
$$

exist and are canonical maps onto $\Omega$; moreover, we assume that the incoming and outgoing momenta satisfy

$$
\theta_{N}^{-}>\cdots>\theta_{1}^{-}, \quad \theta_{N}^{+}<\cdots<\theta_{1}^{+} .
$$

Since the asymptotic Hamiltonians

$$
H^{ \pm} \equiv H \circ \varepsilon_{ \pm}
$$

depend only on $\theta^{ \pm}$, it is easily seen that the choices $H, S_{1}^{\text {out }}, \ldots, S_{N}^{\text {out }}$ and $H, S_{1}^{\text {in }}, \ldots, S_{N}^{\text {in }}$ yield Liouville integrable systems when one defines

$$
\begin{aligned}
S_{k}^{\delta} & \equiv \sum_{1<i_{1}<\cdots<i_{k}<N} \theta_{i_{1}}^{\delta} \cdots \theta_{i_{k}}^{\delta}, \quad \delta=+,- \\
S_{k}^{\text {out }} \text { in } & \equiv S_{k}^{ \pm \circ \mathscr{E}_{ \pm}^{-1} .}
\end{aligned}
$$

Of course, one can choose other functions of $\theta^{ \pm}$that generate the same maximal abelian algebras on $\Omega$. For instance, one could replace $\theta_{i}^{\delta}$ in $(2.14)$ by $\exp \left(\beta \theta_{i}^{\delta}\right)$ with $\beta \in(0, \infty)$. Such choices should not be viewed as different from the previous ones. However, one can just as well introduce new momenta $p\left(\theta^{+}\right)$and canonically conjugate positions $x\left(q^{+}, \theta^{+}\right)$such that the transform of $H$ equals the function $p_{1}$; then the pullbacks to $\Omega$ of the functions $p_{1}, x_{2}, \ldots, x_{N}$ yield a Liouville integrable system that is 
very different from the two previous ones.

This goes to show that any repulsive particle dynamics satisfying the above assumptions gives rise to a plethora of Liouville integrable systems. Let us, therefore, introduce a far stronger notion of integrability (which goes back to [12, p. 339]). We shall say that a dynamics $H$ with the above properties defines a pure soliton system whenever

$$
\theta_{k}^{+}=\theta_{N-k+1}^{-}, k=1, \ldots, N
$$

(conservation of momenta). Notice that this holds if and only if the incoming and outgoing integrals are equal, i.e.,

$$
S_{k}^{\text {in }}=S_{k}^{\text {out }}, k=1, \ldots, N
$$

cf. (2.11)-(2.15).

Pure soliton systems as just defined do exist, and will be studied in Section 2B. However, they are by no means a common occurrence. In fact, within the confines described in the Introduction, the systems considered in Section 2B are the only ones for which the pure soliton property has been proved.

We shall extend the term 'soliton system' to Liouville integrable systems obtained from pure soliton systems $H, S_{1}, \ldots, S_{N}$ by finite-parameter deformations and/or analytic continuation. Admittedly, this sounds like a somewhat loose characterization. However, it does serve to single out the systems studied in Section 2C. Physically speaking, these systems are characterized by attractive and/or confining interactions. In this physical context existence of nontrivial integrals (for the center-of-mass Hamiltonian) is highly exceptional. In fact, the $\mathrm{IV}_{\text {rel }}$ soliton systems defined in Subsection $2 \mathrm{C} 3$ are (to date) the most general Liouville integrable $N$-particle systems for which the interaction depends only on interparticle distances.

\section{B. PURE SOLITON SYSTEMS}

2B1. The Hamittonian H. For the six classes of pure soliton dynamics detailed in this subsection the phase space $\langle\Omega, \omega\rangle$ is given by (2.1), (2.2). The configuration space $G$ can be taken to be

$$
G \equiv\left\{q \in \mathbb{R}^{N} \mid q_{N}<\cdots<q_{1}\right\}
$$

for the Calogero-Moser type systems I, II and

$$
G \equiv \mathbb{R}^{N}
$$

for the nonperiodic Toda type systems VI. For the three nonrelativistic classes the dynamics is of the form

$$
H=\frac{1}{2} \sum_{j=1}^{N} \theta_{j}^{2}+U_{-}(q) .
$$

Specifically, one has

$$
\begin{aligned}
U(q) & \equiv g^{2} \sum_{1<j<k<N} V\left(q_{j}-q_{k}\right), \quad g \in \mathbb{R}^{*} \\
V(x) & \equiv 1 / x^{2} \\
V(x) & \equiv \mu^{2} / 4 \operatorname{sh}^{2} \frac{1}{2} \mu x, \quad \mu \in(0, \infty)
\end{aligned}
$$

Obviously, the $\mathrm{I}_{\mathrm{nr}}$ case arises from the $\mathrm{II}_{\mathrm{nr}}$ case by taking $\mu$ to 0 . Substituting

$$
\begin{aligned}
& q_{j} \rightarrow q_{j}+2 j \mu^{-1} \ln \epsilon \\
& g \rightarrow 1 / \mu \epsilon
\end{aligned}
$$

in the $\mathrm{II}_{\mathrm{nr}}$ Hamiltonian, and taking the strong coupling limit $\epsilon \rightarrow 0$, one obtains the nonperiodic Toda Hamiltonian, for which 


$$
U(q) \equiv \sum_{1<j<N} \exp \left[\mu\left(q_{j}-q_{j-1}\right)\right]
$$

We shall presently discuss the above dynamics and their commuting integrals $S_{1}, \ldots, S_{N}$ in more detail. At this point we only note that among the Hamiltonians in the associated maximal abelian algebras the above Hamiltonians $H$ are singled out not only by their obvious physical interpretation, but also by the fact that their flows are most easily studied. The Hamiltonians that follow now are chosen for similar reasons: Their general structure reads

$$
H \equiv \beta^{-1} \sum_{j=1}^{N} \exp \left(\beta \theta_{j}\right) V_{j}(q)
$$

and the three classes of potentials are given by

$$
\begin{gathered}
V_{j} \equiv \prod_{k \neq j}\left[1+\frac{\beta^{2} g^{2}}{\left(q_{j}-q_{k}\right)^{2}}\right]^{1 / 2}, \quad \beta \in(0, \infty), \quad g \in \mathbf{R}^{*} \\
V_{j} \equiv \prod_{k \neq j}\left[1-\frac{\operatorname{sh}^{2} z}{\operatorname{sh}^{2} \frac{1}{2} \mu\left(q_{j}-q_{k}\right)}\right]^{1 / 2}, \quad \beta, \mu \in(0, \infty), \quad \pm z \in i(0, \pi) \cup\left(\frac{i \pi}{2}+\mathbf{R}\right) \\
V_{j} \equiv f_{T}\left(q_{j+1}-q_{j}\right) f_{T}\left(q_{j}-q_{j-1}\right) \\
f_{T}(x) \equiv\left[1+\beta^{2} \exp (\mu x)\right]^{1 / 2}, \quad \beta, \mu \in(0, \infty) \\
q_{N+1} \equiv-\infty, \quad q_{0} \equiv \infty
\end{gathered}
$$

Note that the restrictions on the parameters guarantee real-valuedness of the Hamiltonians. The subscripts ' $n r^{\prime}$ 'vs. 'rel' refer to the fact that the extra parameter $\beta$ may be viewed as $1 / c$, where $c$ is the speed of light. This is explained in detail in $[1,2,13]$. Here, we only point out that one has

$$
H_{\mathrm{rd}}=N \beta^{-1}+\sum_{j=1}^{N} \theta_{j}+\beta H_{\mathrm{nr}}+O\left(\beta^{2}\right), \quad \beta \rightarrow 0
$$

for the cases I and VI; after the substitution

$$
z \rightarrow \frac{1}{2} i \beta \mu g
$$

this expansion follows for II, as well. Notice that with this substitution one can obtain $H\left(\mathrm{I}_{\mathrm{ret}}\right)$ from $H\left(\mathrm{II}_{\text {rel }}\right)$ by taking $\mu$ to 0 . Moreover, after the substitutions (2.24) and

$$
z \rightarrow \frac{1}{2} i \pi-\ln \epsilon+\ln \beta
$$

one obtains $H\left(\mathrm{VI}_{\mathrm{rel}}\right)$ from $H\left(\mathrm{II}_{\mathrm{rel}}\right)$ by sending $\epsilon$ to 0 .

2B2. The Hamilkonians $S_{1}, \ldots, S_{N}$. For the nonrelativistic dynamics detailed above the existence of integrals was first proved by finding a Lax pair representation

$$
\{H, L\}=[L, M]
$$

for Hamilton's equations. Specifically, one can take as Lax matrices [4]

$$
L\left(\mathrm{II}_{\mathrm{nr}}\right)_{j k}=\delta_{j k} \theta_{k}+i g\left(1-\delta_{j k}\right) \mu / 2 \operatorname{sh} \frac{1}{2} \mu\left(q_{j}-q_{k}\right)
$$

(from which $L\left(\mathrm{I}_{\mathrm{nr}}\right)$ is obvious) and

$$
L\left(\mathrm{VI}_{\mathrm{ar}}\right)_{j k} \equiv \delta_{j k} \theta_{k}+\delta_{j, k-1}+\delta_{j, k+1} \exp \left[\mu\left(q_{j}-q_{j-1}\right)\right]
$$


Then one has in all three cases

$$
H=\frac{1}{2} \operatorname{Tr} L^{2} \text {. }
$$

Thus, it follows from (2.36) that the symmetric functions $S_{1}, \ldots, S_{N}$ of the Lax matrix $L$ are conserved under the $H$ flow. Since the particles repel each other, one also concludes

$$
\begin{array}{ll}
L \rightarrow \operatorname{diag}\left(\theta_{1}^{ \pm}, \ldots, \theta_{N}^{ \pm}\right), & t \rightarrow \pm \infty \\
S_{k} \rightarrow \sum_{1<i_{1}<\cdots<i_{k}<N} \theta_{i_{1}}^{ \pm} \cdots \theta_{i_{k}}^{ \pm}, & t \rightarrow \pm \infty
\end{array}
$$

where $\theta^{ \pm}$denotes the asymptotic momenta. But the spectrum of $L$ is conserved due to (2.36), so that conservation of momenta (2.16) results. Moreover, $\left\{S_{j}, S_{k}\right\}$ is an integral by the Jacobi identity, and it has limit 0 for $t \rightarrow \pm \infty$ since the interparticle distances diverge. Consequently, the Hamiltonians $S_{1}, \ldots, S_{N}$ are in involution.

The arguments just presented are physically convincing, but a rigorous proof of the soliton property involves more work. For the above nonrelativistic systems such a proof was first given by Moser $[14,15]$.

Let us now consider the $\mathrm{II}_{\text {rel }}$ case. Here, it can be shown directly that the functions

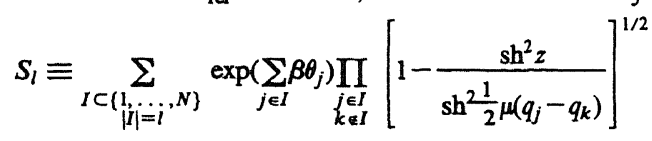

commute with $H=\beta^{-1} S_{1}$. These Hamiltonians are the symmetric functions of the matrix

$$
L\left(\mathrm{II}_{\mathrm{rel}}\right)_{j k} \equiv \exp \left(\beta \theta_{j}\right) V_{j}(q) \operatorname{sh} z / \mathrm{sh}\left(z+\frac{1}{2} \mu\left(q_{j}-q_{k}\right)\right)
$$

(recall (2.29)), as follows from Cauchy's identity. Hence, the spectrum of $L$ is conserved under the flow generated by $H$. Moreover, Moser's argument can be adapted to this flow. This yields (2.40), (2.41) with $\theta_{j}^{\delta}$ replaced by $\exp \left(\beta \theta_{j}^{\delta}\right)$, and entails again involutivity and the soliton property. The same reasoning applies to the $I_{\text {rel }}$ case, obtained by substituting (2.34) in (2.42), (2.43) and taking $\mu$ to 0 . (For more details concerning the assertions in this paragraph we refer to [1].)

For the case $\mathrm{VI}_{\text {rel }}$ a Lax matrix can be obtained from (2.43) by first substituting (2.24) and (2.35) in a suitable similarity transform $\tilde{L}$ of $L\left(\mathrm{II}_{\text {rel }}\right)$ and then taking $\epsilon$ to 0 . Specifically, setting

$$
\tilde{L}_{j k} \equiv \beta^{k-j} \exp \left[\frac{1}{2} \mu\left(q_{j}-q_{k}\right)\right] L\left(\mathrm{II}_{\mathrm{rel}}\right)_{j k}
$$

one obtains

$$
L\left(\mathrm{VI}_{\mathrm{rec}}\right)_{j k}= \begin{cases}0 & k<j-1 \\ \beta^{-1} a_{j} b_{j} & k=j-1 \\ \beta^{k-j} b_{j} & k>j-1\end{cases}
$$

where

$$
\begin{aligned}
& a_{j} \equiv \beta^{2} \exp \left[\mu\left(q_{j}-q_{j-1}\right)\right]\left(1+\beta^{2} \exp \left[\mu\left(q_{j}-q_{j-1}\right)\right]\right]^{-1} \\
& b_{j} \equiv \exp \left(\beta \theta_{j}\right) V_{j}(q)
\end{aligned}
$$

cf. (2.30)-(2.32). The symmetric functions of this matrix read

$$
S_{l}=\sum_{I \subset\{1, j \mid \cdots, N\}} \exp \left(\sum_{j \in I} \beta \theta_{j}\right) \prod_{\substack{j \in I \\ j+1 \in I}} f_{T}\left(q_{j+1}-q_{j}\right) \prod_{\substack{j \in I \\ j \in 1 \notin I}} f_{T}\left(q_{j}-q_{j-1}\right) .
$$

Adapting Moser's argument, the soliton property follows again. (The assertions just made are proved 
in [3]; the opposite ordering is used there.)

With the substitution (2.34) in $L\left(\mathrm{II}_{\mathrm{rel}}\right)$, one easily verifies that

$$
L_{\text {rel }}=1_{N}+\beta L_{\text {nr }}+O\left(\beta^{2}\right), \quad \beta \rightarrow 0
$$

in all three cases. Using this expansion one can obtain the involutivity of the symmetric functions of $L_{\mathrm{nr}}$ as a corollary of the involutivity of the $L_{\mathrm{rel}}$ functions. Specifically, from (2.49) one infers

$$
S_{k, \text { nr }}=\lim _{\beta \rightarrow 0} \beta^{-k} \sum_{l=0}^{k}(-)^{k+l}\left[\begin{array}{c}
N-l \\
N-k
\end{array}\right] S_{l, \text { rel }}
$$

from which the claim readily follows. Note that this formula for $S_{k, n r}$ is far from explicit, in contrast to the formulas (2.42) and (2.48) for $S_{l, \text { rel }}$.

2B3. The action-angle transform and duality. Let us now turn to the construction of an explicit action-angle map for the above systems. We begin by recalling that the Liouville-Amold theorem guarantees the existence of such a diagonalizing map under certain conditions [9]. But these are actually not met for the above systems: The radii of the invariant tori are infinite. Furthermore, even in situations where the intersections of the relevant level sets are compact and connected (as is the case for the type III and IV systems discussed in Section 2C), the Liouville-Arnold theorem is of little help in obtaining explicit information, in much the same way as the spectral theorem is of no use in obtaining the eigenfunctions and spectrum of a concretely given self-adjoint differential operator.

However, it turns out that for the above pure soliton systems and for the related systems of type II and III (cf. Section 2C) one can construct an action-angle map $\Phi$ whose relevant features can be established in much more detail than one might reasonably expect. For all of the pure soliton systems the action-angle phase space can be taken to be

$$
\begin{aligned}
& \hat{\Omega} \equiv\left\{(\hat{q}, \hat{\theta}) \in \mathbf{R}^{2 N} \mid \hat{\theta}_{N}<\cdots<\hat{\theta}_{1}\right\} \\
& \hat{\omega} \equiv \sum_{j=1}^{N} d \hat{q}_{j} \wedge d \hat{\theta}_{j} .
\end{aligned}
$$

Comparing (2.51) with (2.1), (2.18), one sees that $\Omega$ and $\hat{\Omega}$ can be identified in an obvious way for the rational and hyperbolic systems I and II. Doing so, these systems can be defined on $\hat{\Omega}$, too. It now turns out that the inverse $\mathscr{E}$ of $\Phi$ may be viewed as an action-angle map for one of the latter systems. Specifically, the duality thus obtained reads

$$
\mathrm{I}_{\mathrm{nr}} \simeq \hat{\mathrm{I}}_{\mathrm{nr}}, \quad \mathrm{II}_{\mathrm{nr}} \simeq \hat{\mathrm{I}}_{\mathrm{rel}}, \quad \mathrm{I}_{\mathrm{rel}} \simeq \hat{\mathrm{I}}_{\mathrm{nr}}, \quad \mathrm{II}_{\mathrm{rel}} \simeq \hat{\mathrm{I}}_{\mathrm{rel}} .
$$

(The notation will be clear from context.)

Action-angle maps for the systems $\mathrm{VI}_{\mathrm{nr}}$ and $\mathrm{VI}_{\mathrm{rel}}$ can also be constructed explicitly. Again, certain duality properties arise, but these are less useful than (2.53). The maps may be viewed as limits of those of the $\mathrm{II}_{\mathrm{nr}}$ and $\mathrm{II}_{\mathrm{rel}}$ systems, resp. However, this limit is hard to control and yields less information than the direct construction. We refer to [3] for further details on the type VI systems.

The key to the construction of $\Phi$ for the systems of type I and II is a certain commutation relation of the Lax matrix with an auxiliary matrix-valued function $A$ on $\Omega$. We shall now sketch this construction for the $\mathrm{II}_{\mathrm{rel}}$ case, picking $\pm z \in i(0, \pi)$. (The following is taken from our paper [6], where also previous work on special cases [16-18] is discussed.) In this case one has

$$
A_{j k}=\delta_{j k} \exp \left(\mu q_{k}\right)
$$

and the commutation relation reads

$$
\frac{1}{2} \operatorname{cth} z[A, L]=e \otimes e-\frac{1}{2}(A L+L A) .
$$

Here, the vector-valued function $e$ on $\Omega$ is defined by 


$$
e_{j} \equiv \exp \left(\frac{1}{2} \mu q_{j}+\frac{1}{2} \beta \theta_{j}\right) V_{j}(\mu, z ; q)^{1 / 2}
$$

cf. (2.29). Combining this with (2.43), one readily verifies (2.55).

By exploiting (2.55) and Cauchy's identity, it can now be proved that $L$ has positive and simple spectrum on $\Omega$, and that any unitary $U$ such that

$$
U L(\beta, \mu, z ; q, \theta) U^{*}=\operatorname{diag}\left(\exp \left(\beta \hat{\theta}_{1}\right), \ldots, \exp \left(\beta \hat{\theta}_{N}\right)\right), \quad \hat{\theta}_{N}<\cdots<\hat{\theta}_{1}
$$

must satisfy $(U e)_{j} \neq 0, j=1, \ldots, N$. The gauge freedom in the diagonalizing unitary can then be fixed by requiring $(U e)_{j}>0, j=1, \ldots, N$. This entails the existence of a unique vector $\hat{q} \in \mathbb{R}^{N}$ such that

$$
(U e)_{j}=\exp \left(\frac{1}{2} \beta \hat{\theta}_{j}+\frac{1}{2} \mu \hat{q}_{j}\right) V_{j}\left(\beta,-z ; \hat{\theta}^{1 / 2}\right.
$$

and then one obtains the relation

$$
U A(\mu ; q) U^{*}=L(\mu, \beta,-z ; \hat{\theta}, \hat{q}) \equiv \hat{A}(\beta, \mu, z ; \hat{q}, \hat{\theta}) .
$$

As a consequence, a well-defined map

$$
\Phi: \mathbf{\Omega} \rightarrow \hat{\mathbf{\Omega}}, \quad(q, \hat{\theta}) \mapsto(\hat{q}, \hat{\theta})
$$

emerges. From the duality relations (2.58), (2.59) it now readily follows that $\Phi$ is a bijection with inverse

$$
\varepsilon(\beta, \mu, z ; \hat{q}, \hat{\theta})=P \circ \Phi(\mu, \beta,-z ; \hat{\theta}, \hat{q})
$$

where $P$ is the flip map

$$
P(x, y) \equiv(y, x), \quad x, y \in \mathbf{R}^{N} .
$$

Our proof that the bijection $\Phi$ is in fact real-analytic and symplectic is rather long and arduous, and we shall not describe it here. (An important ingredient is scattering theory, to which we shall presently turn.) We finish this subsection by noting that the canonicity of $\Phi$ together with its construction as sketched above entails that $\Phi$ linearizes the flows generated by Hamiltonians of the form

$$
H_{k} \equiv \operatorname{Tr} h\left(\beta^{-1} \ln L\right), \quad h \in C_{\mathbf{R}}^{\infty}(\mathbf{R}) .
$$

Indeed, from (2.57) one has

$$
\left(H_{h}^{\circ} \circ\right)(\hat{q}, \hat{\theta}) \equiv \hat{H}_{h}(\hat{q}, \hat{\theta})=\sum_{j=1}^{N} h\left(\hat{\theta}_{j}\right)
$$

Since $\&$ is canonical, this entails

$$
\exp \left(t H_{h}\right) \circ \varepsilon=\varepsilon \circ \exp \left(t \hat{H}_{h}\right)
$$

Hence, the nonlinear flow

$$
(q, \theta) \mapsto \exp \left(t H_{h}\right)(q, \theta) \equiv(q(t), \theta(t))
$$

maps into the linear flow

$$
(\hat{q}, \hat{\theta}) \mapsto \exp \left(t \hat{H}_{h}\right)(\hat{q}, \hat{\theta})=\left(\hat{q}_{1}+t h^{\prime}\left(\hat{\theta}_{1}\right), \ldots, \hat{q}_{N}+t h^{\prime}\left(\hat{\theta}_{N}\right), \hat{\theta}\right) \equiv(\hat{q}(t), \hat{\theta})
$$

as advertised.

2B4. Soliton scattering. As we have just seen, one has

$$
(q(t), \theta(t))=\varepsilon(\hat{q}(t), \hat{\theta})
$$

for Hamiltonians of the form (2.63). For the $\mathrm{II}_{\mathrm{rel}}$ systems the quantities $\exp \left(\mu q_{j}(t)\right)$ are the (ordered) eigenvalues of the matrix 


$$
\hat{A}\left(\beta, \mu, z ; \hat{q}_{1}+t h^{\prime}\left(\hat{\theta}_{1}\right), \ldots, \hat{q}_{N}+t h^{\prime}\left(\hat{\theta}_{N}\right), \hat{\theta}\right)
$$

cf. (2.59). Hence, the long-time asymptotics of $q_{j}(t)$ boils down to a problem of spectral asymptotics. Specifically, assuming henceforth

$$
h^{\prime \prime}(x)>0, \quad \forall x \in \mathbb{R}
$$

one needs the spectral asymptotics of matrices of the form $M \exp (t D)$, where $M$ is positive and $D$ is of the form

$$
D=\operatorname{diag}\left(d_{1}, \ldots, d_{N}\right), \quad d_{N}<\cdots<d_{1} .
$$

This problem can be solved under less restrictive assumptions on $M$ and $D$, and the generalization thus obtained is essential in our proof that $\Phi$ is canonical. (We mention in passing that the set of matrices $M$ that are allowed (cf. [6, p. 157]) may be viewed as the big cell in the Bruhat decomposition of $G L(N, \mathrm{C})$. This observation is possibly useful in tackling other root systems. However, the use of upper/lower factorization appears not to simplify the proof of 1.c. Theorem A2.)

As a consequence of the spectral analysis just referred to, it can be concluded that the assumption (2.70) entails

$$
\begin{array}{ll}
q_{N-j+1}(t)-\hat{q}_{j}(t) \rightarrow \mp \frac{1}{2} \Delta_{j}(\hat{\theta}), & t \rightarrow \pm \infty \\
\theta_{N-j+1}(t)-\hat{\theta}_{j} \rightarrow 0 & t \rightarrow \pm \infty
\end{array}
$$

uniformly on compacts. Here, one has

$$
\begin{aligned}
& \Delta_{j}(\theta) \equiv \sum_{k<j} \delta\left(\theta_{j}-\theta_{k}\right)-\sum_{k>j} \delta\left(\theta_{j}-\theta_{k}\right) \\
& \delta(\theta) \equiv \mu^{-1} \ln \left(1-\operatorname{sh}^{2} z / \mathrm{sh}^{2} \frac{1}{2} \beta \theta\right) .
\end{aligned}
$$

Thus, the scattering map

$$
S: \Omega^{-} \equiv\left\{\left(q^{-}, \theta^{-}\right) \in \mathbf{R}^{2 N} \mid \theta_{N}^{-}>\cdots>\theta_{1}^{-}\right\} \rightarrow \Omega^{+} \simeq \hat{\Omega}
$$

is given by

$$
S\left(q_{1}^{-}, \ldots, q_{\bar{N}}, \theta_{1}^{-}, \ldots, \theta_{\bar{N}}^{-}\right)=\left(q_{\bar{N}}^{-}+\Delta_{N}\left(\theta^{-}\right), \ldots, q_{1}^{-}+\Delta_{1}\left(\theta^{-}\right), \theta_{\bar{N}}^{-}, \ldots, \theta_{1}^{-}\right) .
$$

The factorization into two-particle $S$-maps exhibited by (2.74), (2.77) can be understood heuristically as a consequence of the conservation of momenta (2.16) already obtained in Subsection 2B2 (cf. [19] and $[1$, p. 381] for this argument).

The $S$-map (2.77) is shared not only by all dynamics $H_{h}$ with $h$ satisfying (2.70), but also by a much larger class of dynamics, containing in particular $S_{1}, \ldots, S_{N-1}$. (Note only $S_{1}$ is of the form $H_{h}$, with $h(x)=\exp (\beta x)$.) In fact, this invariance principle can be further generalized, and may then be viewed as an asymptotic constancy property of the canonical transformation $\varepsilon$, cf. [6, Th. 5.1]. By duality, $\Phi=\mathcal{E}^{-1}$ also has this property. Conversely, any map of this kind gives rise to a duality between pure soliton systems. Are the $\mathrm{II}_{\mathrm{rel}}$ maps the most general real-analytic and symplectic bijections between $\langle\Omega, \omega\rangle$ and $\langle\hat{\Omega}, \hat{\omega}\rangle$ having both the duality $(\simeq$ involution) and the asymptotic constancy properties?

2C. SYSTEMS RELATED TO PURE SOLITON SYSTEMS

2C1. Systems with solitons and antisolitons. Following Calogero [20], let us substitute

$$
q_{j} \rightarrow q_{j}+i \pi / \mu, \quad j=n+1, \ldots, N
$$

(with $1 \leqslant n \leqslant N-1$ ) in the $\mathrm{II}_{\mathrm{nr}}$ Hamiltonian. This yields 
$H=\frac{1}{2} \sum_{j=1}^{N} \theta_{j}^{2}+\frac{1}{4} g^{2} \mu^{2}\left[\sum_{\substack{1<j<k<n \\ n+1<j<k<N}} \frac{1}{\operatorname{sh}^{2} \frac{1}{2} \mu\left(q_{j}-q_{k}\right)}-\sum_{\substack{1<j<n \\ n+1<k<N}} \frac{1}{\operatorname{ch}^{2} \frac{1}{2} \mu\left(q_{j}-q_{k}\right)}\right] \quad\left(\tilde{I}_{n r}\right)$.

Thus, the resulting systems may be viewed as nonrelativistic interacting particle systems with $n$ particles of positive charge (solitons) and $N-n$ particles of negative charge (antisolitons); particles with the same/opposite charge repel/attract each other.

If we make the substitution (2.78) in the more general $\mathbb{I}_{\text {rel }}$ dynamics (2.27), (2.29), then we obtain a real-valued smooth Hamiltonian on

$$
\Omega^{(n)} \equiv\left\{(q, \theta) \in \mathbb{R}^{2 N} \mid q_{N}<\cdots<q_{n+1}, q_{n}<\cdots<q_{1}\right\}
$$

provided $z \in i(0, \pi / 2)$. We shall assume this for the moment, and will presently return to the critical value $z \equiv i \pi / 2$. As it turns out, the substitution (2.78) leads to the same picture as before. The physical characteristics of both the $\tilde{\mathrm{I}}_{\mathrm{ar}}$ and the $\tilde{\mathrm{I}}_{\text {rel }}$ systems can be completely elucidated via an explicit action-angle map $\Phi^{(n)}$. (The results to be summarized now are detailed in [7].) Again, the commutation relation (2.55) is the key to the construction of $\Phi^{(n)}$. The substitution (2.78) gives rise to a Lax matrix that is pseudo-self-adjoint w.r.t. the indefinite metric $\operatorname{diag}\left(\mathbb{1}_{n},-\mathbb{1}_{N-n}\right)$. By exploiting wellknown properties of such matrices and Cauchy's identity, one can show that the spectrum of $L$ lies in the closure of a strip

$$
S_{\alpha} \equiv\{w \in \mathbb{C}|||m w|<\alpha\}
$$

for the $\tilde{\mathrm{II}}_{\mathrm{nr}}$ case, and in a sector $\exp \left(\bar{S}_{\alpha}\right)$ for the $\tilde{\mathrm{I}}_{\mathrm{rel}}$ case. Specifically, one has

$$
\begin{array}{ll}
\alpha=\frac{1}{2}|\mu g| & \left(\tilde{\mathrm{I}}_{\mathrm{ar}}\right) \\
\alpha=|z| & \left(\tilde{\mathrm{II}}_{\mathrm{rel}}\right) .
\end{array}
$$

Non-real eigenvalues occur in complex-conjugate pairs, and each such pair corresponds to a solitonantisoliton bound state. All channels one can envisage occur, but they do not couple. That is, there is a region in $\Omega^{(n)}$ where $L$ has real and simple spectrum, corresponding to an asymptotics of freely moving solitons and antisolitons, and there are regions with $k \leqslant \min (n, N-n)$ bound pairs. There also exist points in $\Omega^{(n)}$ for which $L$ is not diagonalizable; such points do not behave as scattering states. Moreover, there are points that behave as multi-body bound states; this phenomenon depends on the dynamics one chooses.

The case $z=i \pi / 2$ is different only in as much as singularities occur when soliton and antisoliton positions coincide. Technically speaking, this entails that the $H$ flow (for instance) is not complete. However, the action-angle map enables one to define the flow for all times in a natural way, but for a discrete set of times where solitons 'overtake' antisolitons. The singular character of $z=i \pi / 2$ is most easily seen for $N=2$. Then $H$ can be written

$$
H=2 \beta^{-1} \exp \left[\frac{1}{2} \beta\left(\theta_{1}+\theta_{2}\right)\right] \operatorname{ch} \frac{1}{2} \beta\left(\theta_{1}-\theta_{2}\right)\left|\operatorname{th} \frac{1}{2} \mu\left(q_{1}-q_{2}\right)\right| .
$$

Thus, the set $q_{1}-q_{2}$ plays a special role. In particular, by energy conservation $\theta_{1}-\theta_{2}$ diverges for $q_{1}-q_{2} \rightarrow 0$.

Instead of substituting (2.78), one might also substitute

$$
\theta_{j} \rightarrow \theta_{j}+i \pi / \beta, \quad j=n+1, \ldots, N
$$

in the $\mathrm{II}_{\text {rel }}$ Lax matrix (2.43). Indeed, this also yields real-valued symmetric functions, cf. (2.42). This type of Lax matrix actually arises on the subset of the action-angle phase space corresponding to the 'no bound state' subset $\Omega_{0}^{(n)} \subset \Omega^{(n)}$. (This fact can be understood from the self-duality of the $\mathrm{II}_{\text {rel }}$ regime described in Subsection 2B3.) As a consequence, one can infer that the $H$ flow arising via (2.85) is not complete. Indeed, the corresponding dual flow on the subset of $\hat{\Omega}^{(n)}$ just mentioned pulls 
back to a linear flow on $\Omega^{(n)}$; for an open set of initial points this flow leaves $\Omega_{0}^{(n)}$ in finite time. This unstable behaviour can be physically understood by noting that $(2.85)$ amounts to flipping the sign of the particle mass, cf. (2.27).

One can also continue both some $q_{j}$ and some $\theta_{j}$, since this still leads to real-valued commuting Hamiltonians, cf. (2.42). However, in this case the Lax matrix appears not to have any properties that can be used to elucidate the relevant features of the dynamics.

As a preparation for the next subsection, we now rephrase the mathematical setting of the above results. We have at our disposal a symplectic diffeomorphism $\Phi$ from $\langle\Omega, \omega\rangle$ (cf. (2.1), (2.2), (2.18)) onto $\langle\hat{\Omega}, \hat{\omega}\rangle$ (cf. $(2.51)$, (2.52)), which is real-analytic in the parameters and in the canonical coordinates, cf. [6, App. B,C]. This map can be analytically continued in $q$ and then restricted to certain real, $N$-dimensional submanifolds of $\mathbb{C}^{N}$, cf. (2.78). These give rise to new phase spaces (cf. (2.80)), whose images under $\Phi$ can be explicitly determined. Correspondingly, one can obtain detailed information concerning associated Hamiltonian flows.

More generally, singularity and monodromy properties of $\Phi$ can be determined rather explicitly, but the structure of the image set appears too complicated to answer with ease every question one may care to ask. For instance, the behaviour under the continuation (2.85) can be understood from duality considerations, but we have no information concerning simultaneous continuation in $q$ and $\theta$. The feasibility of answering concrete questions hinges on special features of the Lax matrix, restricted to the real, $2 N$-dimensional submanifold of $C^{2 N}$ which is chosen as a new phase space.

2C2. Sutherland type systems and their duals. The preceding considerations suggest a systematic approach: Find the physically interesting and mathematically manageable phase spaces embedded in $\mathbb{C}^{2 N}$, as obtained from the pure soliton regimes via analytic continuation in $q, \theta$ and/or the parameters.

As a first example along these lines, one may start from the $\mathrm{II}_{\mathrm{nr}}$ systems and continue the positive parameter $\mu$ to the imaginary axis. (Equivalently, one may keep $\mu$ positive and take $g$ and $q_{1}, \ldots, q_{N}$ purely imaginary.) Then one obtains again a self-adjoint Lax matrix, with

$$
H \equiv \frac{1}{2} \operatorname{Tr} L^{2}=\frac{1}{2} \sum_{j=1}^{N} \theta_{j}^{2}+\frac{1}{4} g^{2}|\mu|^{2} \sum_{1<j<k<N} \frac{1}{\sin ^{2} \frac{1}{2}|\mu|\left(q_{j}-q_{k}\right)} \quad\left(\mathrm{III}_{\mathrm{nr}}\right) .
$$

The obvious physical interpretation of the $\mathrm{III}_{\mathrm{nr}}$ systems thus obtained is now in terms of particles on a ring. On account of energy conservation the angular distances $\left|q_{j}-q_{k}\right|$ remain finite for all times. Omitting the center-of-mass motion, an appropriate configuration space is the 'Weyl alcove'

$$
G \equiv\left\{x \in \mathbb{R}^{N-1}\left|x_{1}, \ldots, x_{N-1}>0, \sum_{j=1}^{N-1} x_{j}<2 \pi /\right| \mu \mid\right\}, x_{j} \equiv q_{j}-q_{j+1}
$$

and each state in the corresponding $(2 N-2)$-dimensional phase space is a bound state.

The action-angle map $\Phi$ can be determined explicitly for the III $_{n r}$ systems [8]. In particular, one can prove that the (ordered) eigenvalues of $L\left(\mathrm{III}_{\mathrm{nr}}\right)$ satisfy

$$
\hat{\theta}_{j}-\hat{\theta}_{j+1} \geqslant|\mu g| \text {. }
$$

The exceptional set $\Omega_{e}$ where one or more equality signs are realized is nowhere dense and has. measure zero; it contains the equilibrium configuration

$$
q_{j}=\frac{\pi}{N|\mu|}(N+1-2 j), \quad \theta_{j}=0, \quad j=1, \ldots, N
$$

for which all equality signs in (2.88) apply. The map $\Phi$ is defined on the complement of $\Omega_{e}$, and sets up a duality with $\hat{I I I}_{\text {nr }}$ systems that can be obtained from the above $\mathrm{I}_{\text {rel }}$ systems, as follows:

$$
\mathrm{I}_{\mathrm{rel}} \rightarrow \mathrm{III}_{\mathrm{nr}}: \beta, q, \theta \rightarrow \mu, \hat{\theta}, \hat{q}, \quad \mu \in i(0, \infty) .
$$


At first sight the $\hat{I I I}_{n x}$ systems thus obtained appear unphysical. Indeed, due to $(2.88)$ the $\hat{\theta}$ dependent potentials in $S_{1}, \ldots, \hat{S}_{N}$ are positive on $\Omega\left(\mathrm{III}_{\mathrm{nr}}\right)$, but the exponentials in (the $\mathrm{I}_{\text {rel }}$ specialization of) (2.42) turn into phase factors after the substitution (2.90). However, this is easily remedied. What matters is whether there exists a real form for the maximal abelian Poisson algebra. To explain that this is indeed the case, we need a piece of information from [1,2] we had no occasion to mention so far. This is the fact that the functions $S_{l}(\beta)$ given by $(2.42)$ commute with $S_{k}(-\beta)$. Therefore, we may replace exp by ch at the rhs of (2.42), and then we do get real-valued $\hat{q}$-dependence under the substitution (2.90).

In particular, the dynamics

$$
\hat{H} \equiv \beta^{-1} \sum_{j=1}^{N} \cos \left(|\mu| \hat{q}_{j}\right) \prod_{k \neq j}\left[1-\frac{|\mu g|^{2}}{\left(\hat{\theta}_{j}-\hat{\theta}_{k}\right)^{2}}\right]^{1 / 2} \quad\left(\hat{\mathrm{II}}_{\mathrm{nr}}\right)
$$

is real-valued. The flow it generates has a quite different character compared to the flow generated by (2.86). In fact, the $\hat{I I I}_{\mathrm{nr}}$ systems may be viewed as pure soliton systems, in a sense detailed in [8].

As a second example of the program described in the first paragraph of this subsection, one may continue the parameter $g$ in the $\mathrm{II}_{\mathrm{nr}}$ systems to the imaginary axis. (In fact, this can already be done for the special case $I_{n r}$.) However, this leads to 'physically interesting' systems that seem not to be 'mathematically manageable'.

In the same spirit one can obtain from the $\mathrm{II}_{\mathrm{rel}}$ pure soliton systems seven other regimes of physical interest, by first parametrizing $z$ as

$$
z=\frac{1}{2} i \beta \mu g, \quad \beta, \mu, g \in(0, \infty) \quad\left(\mathrm{II}_{\mathrm{rel}}\right)
$$

and then taking $\beta, \mu$ and/or $g$ purely imaginary. However, we can only handle the three cases that arise when one keeps $g$ real, for reasons just mentioned. The systems obtained by taking $\mu \in i(0, \infty) / \beta, \mu \in i(0, \infty)$ will be denoted $\mathrm{III}_{\text {rel }} / \mathrm{III}_{\text {rel, } b}$. When one only takes $\beta \in i(0, \infty)$, one obtains systems that may be viewed as the $\hat{\mathrm{III}} \mathrm{rel}_{\text {rel }}$ systems (i.e., the systems dual to the $\mathrm{III}_{\text {rel }}$ systems, cf. also (2.90)).

The salient features of the $\mathrm{III}_{\text {rel }}$ and $\mathrm{III}_{\text {rel }}$ systems are essentially the same as those of the $\mathrm{III}_{\mathrm{nr}}$ and $\hat{I I I}_{\mathrm{nr}}$ cases already described. In particular, with (2.34) in force, one can prove the inequalities (2.88) for the eigenvalues $\exp \left(\beta \hat{\theta}_{j}\right)$ of $L\left(\mathrm{III}_{\mathrm{rel}}\right)$, and for the equilibrium (2.89) one again obtains equality signs.

The self-dual regime $\mathrm{III}_{\mathrm{rel}, b}$ is quite different from all previous ones. As before, we should replace exp by ch in (2.42) to obtain real-valued dependence on $\theta$. However, to also ensure real-valued $q$ dependence, one now needs a center-of-mass configuration space

$$
G_{b} \equiv\left\{x \in \mathbb{R}^{N-1}\left|x_{1}, \ldots, x_{N-1}>\right| \beta g\left|, \sum_{j=1}^{N-1} x_{j}<2 \pi /\right| \mu|-| \beta g \mid\right\}, \quad x_{j} \equiv q_{j}-q_{j+1} .
$$

As a consequence, $G_{b}$ is non-empty if and only if

$$
N<i \pi / z, \quad z=\frac{1}{2} i \beta \mu g \in i\left(0, \frac{1}{2} \pi\right) \text {. }
$$

Therefore, not only the configuration space, but also the allowed particle number is bounded. In fact, the regime III $_{\text {rel, } b}$ has yet a third boundedness property: The natural center-of-mass phase space is not $T^{*} G \simeq G \times \mathbb{R}^{N-1}$, but rather $G \times U(1)^{N-1}[8]$.

2C3. Elliptic systems. The nonrelativistic Hamiltonians considered thus far may all be regarded as special cases of

$$
H=\frac{1}{2} \sum_{j=1}^{N} \theta_{j}^{2}+g^{2} \sum_{1<j<k<N} \mathscr{P}\left(q_{j}-q_{k} ; \omega, \omega^{\prime}\right), \quad g \in \mathbb{R}^{*}, \quad \omega,-i \omega^{\prime} \in(0, \infty) \quad\left(\mathrm{IV}_{\mathrm{nr}}\right)
$$


(Here, $\mathscr{P}$ is the Weierstrass $P$-function; the restriction on the primitive periods $2 \omega, 2 \omega^{\prime}$ guarantees positivity on R.) More precisely, this is true for the $\mathrm{I}_{\mathrm{nr}}, \mathrm{II}_{\mathrm{nr}}, \mathrm{II}_{\mathrm{nr}}$ and $\mathrm{VI}_{\mathrm{nr}}$ Hamiltonians; one needs to shift $q_{n+1}, \ldots, q_{N}$ over $\omega^{\prime}$ (obtaining a real-valued $\tilde{\mathrm{I}}_{\mathrm{ar}}$ Hamiltonian) to ensure that the $\tilde{\mathrm{I}}_{\mathrm{nr}}$ Hamiltonian (2.79) results (up to a constant) when one sets $\omega^{\prime}=i \pi / \mu$ and takes $\omega$ to $\infty$.

The commuting Hamiltonians that generalize those of previous sections can be taken to be the symmetric functions of the matrix

$$
L\left(\mathrm{IV}_{\mathrm{nr}}\right)_{j k}=\delta_{j k} \theta_{k}+i g\left(1-\delta_{j k}\right) \frac{\sigma\left(q_{j}-q_{k}+\lambda\right)}{\sigma(\lambda) \sigma\left(q_{j}-q_{k}\right)} .
$$

Here, $\sigma$ is the Weierstrass $\sigma$-function and $\lambda \in \mathbb{C}$ an auxiliary parameter. The Lax matrix (2.96) was introduced by Krichever [21]. He shows that the $H$ flow linearizes on the Jacobian of the genus $N$ curve defined by

$$
\left|L(\lambda)+\boldsymbol{\alpha} \mathbb{1}_{N}\right|=0
$$

Krichever's results pertain to complex $q_{j}$ and $\theta_{j}$, and it seems not an easy matter to obtain results for real $2 N$-dimensional phase spaces of physical interest.

As the relativistic generalization of $(2.95)$ one can take [1,2] the dynamics (2.27), with

$$
\begin{aligned}
& V_{j} \equiv \prod_{k \neq j} f\left(q_{j}-q_{k}\right) \\
& f^{2}(x) \equiv \sigma^{2}(\gamma)[\mathscr{P}(\gamma)-\mathscr{P}(x)], \quad \pm i \gamma \in\left(0,-2 i \omega^{\prime}\right) \quad\left(\mathrm{IV}_{\mathrm{rel}}\right) .
\end{aligned}
$$

Then one has $H=\beta^{-1} \operatorname{Tr} L$, where $L$ is the Lax matrix [22, 2]

$$
L\left(\mathrm{IV}_{\mathrm{rel}}\right)_{j k} \equiv \exp \left(\beta \theta_{j}\right) V_{j}(q) \frac{\sigma\left(q_{j}-q_{k}+\lambda\right)}{\sigma(\lambda)} \frac{\sigma(\gamma)}{\sigma\left(q_{j}-q_{k}+\gamma\right)}
$$

A Lax pair formulation for the $H$ flow has been found by Bruschi and Calogero [22], whereas in [2] it is proved that the symmetric functions of $L$ are given by

$$
\Sigma_{l}=\frac{\sigma(\lambda-\gamma)^{l-1} \sigma(\lambda+(l-1) \gamma)}{\sigma(\lambda)^{l}} \sum_{I \subset\{1, \ldots \ldots, N\}} \exp \left(\sum_{j \in I} \beta \theta_{j}\right) \prod_{\substack{j \in I \\ k \notin \mid=I}} f\left(q_{j}-q_{k}\right)
$$

and are in involution. Note that after the substitution

$$
\gamma \rightarrow i \beta g
$$

the expansion (2.49) again holds true. Therefore, involutivity of the symmetric functions $S_{k}$ of $L\left(\mathrm{IV}_{\mathrm{nr}}\right)$ follows as a corollary, cf. (2.50). Moreover, since $\Sigma_{l}$ depends on $\lambda$ only via a multiplicative factor, the functions $S_{k}\left(\lambda_{1}\right)$ and $S_{l}\left(\lambda_{2}\right)$ also commute pairwise when $\lambda_{1} \neq \lambda_{2}$.

For the $I V_{\text {rel }}$ case the curve (2.97) appears to have (generically) genus $1+N(N-1) / 2[2]$.

There is yet another parameter regime of physical interest, which generalizes the $\mathrm{III}_{\mathrm{rel}, b}$ regime. It is defined by taking

$$
\beta \in i \mathbf{R}^{*}, \quad \gamma \in(0, \omega) \quad\left(\mathrm{IV}_{\mathrm{rel}, b}\right) .
$$

The generalizations of (2.93) and (2.94) read

$$
\begin{aligned}
& G_{b} \equiv\left\{x \in \mathbb{R}^{N-1} \mid x_{1}, \ldots, x_{N-1}>\gamma, \sum_{j=1}^{N-1} x_{j}<2 \omega-\gamma\right\}, x_{j} \equiv q_{j}-q_{j+1} \\
& N<2 \omega / \gamma
\end{aligned}
$$

and commuting real-valued Hamiltonians can then be defined via (2.101), with the prefactors omitted and exp replaced by $\mathrm{ch}$.

2C4. Periodic Toda type systems. The periodic Toda systems can be defined by 


$$
\begin{aligned}
& H=\frac{1}{2} \sum_{j=1}^{N} \theta_{j}^{2}+\tau^{2} \sum_{1<j<N} \exp \left[\mu\left(q_{j}-q_{j-1}\right)\right]+\tau^{2} \exp \left[\mu\left(q_{1}-q_{N}\right)\right] \\
& \tau \in \mathbb{R}^{*}, \quad \mu \in(0, \infty)
\end{aligned}
$$

(The systems denoted type $\mathrm{V}$ by Olshanetsky and Perelomov $[4,5]$ may be viewed as type I systems perturbed by an external field.) As Lax matrix one can take [23,4]

$$
\begin{aligned}
& L\left(\mathrm{~V}_{\mathrm{nr}}\right)_{j k}=\delta_{j k} \theta_{k}+\delta_{j, k-1}+\tau^{2} \delta_{j, k+1} \exp \left[\mu\left(q_{j}-q_{j-1}\right)\right] \\
& -(i \tau)^{N} \lambda \delta_{j N} \delta_{k 1}-\tau^{2}(i \tau)^{-N} \lambda^{-1} \delta_{j 1} \delta_{k N} \exp \left[\mu\left(q_{1}-q_{N}\right)\right], \quad \lambda \in \mathbb{C}^{*}
\end{aligned}
$$

and then (2.39) again holds true (up to a constant when $N=2$ ). Substituting

$$
q_{j} \rightarrow q_{j}-2 j \mu^{-1} \ln \tau, \quad j=1, \ldots, N
$$

one obtains $L\left(\mathrm{VI}_{\mathrm{ar}}\right)$ in the weak coupling limit $\tau \rightarrow 0$, cf. (2.38).

The commuting $V_{n r}$ flows can be linearized on the Jacobian of the algebraic curve (2.97), which is hyperelliptic and has (generically) genus $N-1[24,25]$. Just as for the $\mathrm{IV}_{\mathrm{nr}}$ case, little seems to be known for real $q$ and $\theta$.

The $V_{\text {rel }}$ generalization of $(2.106)$ can be taken to be (2.27) with $V_{j}$ given by (2.30), but now (2.31), (2.32) should be replaced by

$$
\begin{aligned}
& f_{\tau}(x) \equiv\left[1+\tau^{2} \beta^{2} \exp (\mu x)\right]^{1 / 2} \\
& q_{N+1} \equiv q_{1}, \quad q_{0} \equiv q_{N}
\end{aligned}
$$

This again entails the expansion (2.33). The commuting integrals are still given by (2.48), but now with (2.109), (2.110) in force [3]. Bruschi and Ragnisco [26] found various Lax pair formulations for the $H$ flow, and used one of these to obtain a linearization on a spectral curve defined in analogy with (2.97).

Inozemtsev recently observed that the $\mathrm{V}_{\mathrm{nr}}$ Hamiltonian (2.106) may be obtained as a strong coupling limit of the $\mathrm{IV}_{\mathrm{nr}}$ Hamiltonian (2.95) [27]. A priori, his argument sheds no light on the behaviour of the conserved quantities in his limit. However, we shall now show how one can obtain the $V_{n r}$ Lax matrix (2.107) from the $I V_{n r}$ Lax matrix (2.96). Moreover, we shall obtain a $V_{\text {rel }}$ Lax matrix from the $\mathrm{IV}_{\mathrm{re}} \mathrm{Lax}$ matrix (2.100). On account of the mode of convergence and the argument embodied in (2.50), the Liouville integrability of the systems of type $\mathrm{V}$ may then be viewed as a corollary of the involutivity of the functions $\Sigma_{k}$ given by (2.101).

The limits to be detailed now can be easily controlled and verified by using the representation

$\sigma(q ; \omega, i \pi / \mu)=\exp \left(\eta^{\prime} \mu q^{2} / 2 i \pi\right) \frac{2}{\mu} \operatorname{sh} \frac{1}{2} \mu q \prod_{k>0} \frac{[1-\exp (\mu q-2 k \mu \omega)][1-\exp (-\mu q-2 k \mu \omega)]}{[1-\exp (-2 k \mu \omega)]^{2}}$

for the Weierstrass $\sigma$-function. (Here, the notation of [28] is used.) For both limits one needs position shifts given by

$$
q_{j} \rightarrow q_{j}-2 j \omega / N, \quad j=1, \ldots, N
$$

The first-mentioned connection can be made by starting from the similarity transform

$$
\tilde{L}_{j k} \equiv L\left(\mathrm{IV}_{\mathrm{nr}}\right)_{j k} \exp \left[\frac{i}{\pi} \eta^{\prime} \mu \lambda\left(q_{j}-q_{k}\right)+(j-k) \frac{\mu \omega}{N}\right]
$$

Substituting (2.112) in $\tilde{L}$, together with

$$
\begin{aligned}
& g \rightarrow \tau \mu^{-1} \exp (\mu \omega / N) \\
& \lambda \rightarrow \omega+\frac{i \pi}{\mu}-\frac{1}{\mu} \ln \lambda
\end{aligned}
$$

one obtains 


$$
\lim _{\omega \rightarrow \infty} \tilde{L}_{j k}=L\left(\mathrm{~V}_{\mathrm{nr}}\right)_{j k}(i \tau)^{j-k}
$$

(as anticipated in the choice of spectral parameter in (2.107)).

For the second transition we make use of a renormalized Lax matrix

$$
L_{j k}^{r} \equiv L\left(\mathrm{IV}_{\mathrm{rel}}\right)_{j k} \exp \left(\frac{i}{\pi} \eta^{\prime} \mu\left[\frac{1}{2} \gamma^{2}(N-1)+(\lambda-\gamma)\left(q_{j}-q_{k}\right)\right]\right)
$$

Substituting (2.112) in $L^{r}$, together with

$$
\begin{aligned}
& \gamma \rightarrow \frac{2 \omega}{N}+\frac{i \pi}{\mu}+\frac{2}{\mu} \ln (\beta \tau) \\
& \lambda \rightarrow \frac{2 \omega}{N}+\frac{2}{\mu} \ln (\beta \tau)-\frac{N}{\mu} \ln (i \beta \tau)-\frac{1}{\mu} \ln \lambda
\end{aligned}
$$

one obtains

$$
\begin{aligned}
& \lim _{\omega \rightarrow \infty} L^{r}=B(A+\Lambda) \equiv L\left(\mathrm{~V}_{\mathrm{rel}}\right) \\
& B \equiv \operatorname{diag}\left(b_{1}, \ldots, b_{N}\right) \\
& A_{j k} \equiv \delta_{j, k+1}\left[1+(i \beta \tau)^{N} \lambda\right] a_{j}-\delta_{j 1} \delta_{k N}\left[1+(i \beta \tau)^{-N} \lambda^{-1}\right] a_{1} \\
& \Lambda_{j k} \equiv \begin{cases}-(i \beta \tau)^{N} \lambda & k \leqslant j-1 \\
1 & k>j-1\end{cases} \\
& a_{j} \equiv \beta^{2} \tau^{2} \exp \left[\mu\left(q_{j}-q_{j-1}\right)\right]\left(1+\beta^{2} \tau^{2} \exp \left[\mu\left(q_{j}-q_{j-1}\right)\right]\right)^{-1} \\
& b_{j} \equiv \exp \left(\beta \theta_{j}\right)\left(1+\beta^{2} \tau^{2} \exp \left[\mu\left(q_{j+1}-q_{j}\right)\right]\right]^{1 / 2}\left(1+\beta^{2} \tau^{2} \exp \left[\mu\left(q_{j}-q_{j-1}\right)\right]\right)^{1 / 2}
\end{aligned}
$$

Taking $\omega \rightarrow \infty$ in the symmetric functions of $L^{r}$ (which follow from (2.101)) one obtains the symmetric functions of $L\left(\mathrm{~V}_{\text {rel }}\right)$. Explicitly, one finds

$$
\Sigma_{l}= \begin{cases}{\left[1+(i \beta \tau)^{N} \lambda\right]^{y-1} S_{l},} & l=1, \ldots, N-1 \\ {\left[1+(i \beta \tau)^{N} \lambda\right]^{N-1}\left[1+(i \beta \tau)^{N} \lambda^{-1}\right] S_{N},} & l=N\end{cases}
$$

where $S_{1}, \ldots, S_{N}$ are given by (2.48) (with (2.109), (2.110) in force, of course). Thus, involutivity of $S_{1}, \ldots, S_{N}$ may be viewed as a corollary of the involutivity of the elliptic Hamiltonians (2.101), as announced above.

We add four more remarks. First, up to a constant and a similarity transformation, the matrix $L\left(V_{\text {rel }}\right)^{T}$ equals the Lax matrix of a Lax pair for the $H$ flow that was recently obtained by Oevel and Ragnisco [29]. Secondly, setting

$$
\tilde{L}\left(\mathrm{~V}_{\mathrm{rel}}\right)_{j k} \equiv L\left(\mathrm{~V}_{\mathrm{rel}}\right)_{j k} \beta^{k-j}
$$

and substituting (2.108), one obtains

$$
\lim _{\mathrm{r} \rightarrow 0} \tilde{L}\left(\mathrm{~V}_{\mathrm{rel}}\right)=L\left(\mathrm{VI}_{\mathrm{rel}}\right)
$$

cf. (2.45)-(2.47). Thirdly, one has

$$
\tilde{L}\left(\mathrm{~V}_{\mathrm{rel}}\right)=\mathbf{1}_{N}+\beta L\left(\mathrm{~V}_{\mathrm{nr}}\right)+O\left(\beta^{2}\right), \quad \beta \rightarrow 0
$$

cf. (2.107). Therefore, the Liouville integrability of the nonrelativistic periodic Toda systems may be viewed as a corollary of the Liouville integrability of our relativistic generalizations, cf. (2.49), (2.50). Fourthly, from (2.129) and (2.126) one infers (by using (2.50) with $S_{l, \text { red }}$ replaced by $\Sigma_{l}$ ) that the functions $S_{k}\left(V_{\mathrm{nr}}\right)$ are $\lambda$-independent for $k<N$, whereas for $k=N$ the $\lambda$-dependence is given by an additive term $(i \tau)^{N}\left[\lambda^{-1}+(-)^{N} \lambda\right]$. (This can also be seen directly from (2.107).) 


\section{QUANTUM SOLTON SYSTEMS}

\section{A. QUANTUM INTEGRABIITTY AND THE SOLITON PROPERTY}

A Hamiltonian on a symplectic manifold $\langle\Omega, \omega\rangle$ does not have any (nontrivial) integrals, in general. Therefore, the notion of Liouville integrability is a restrictive and hence useful one in the context of general classical Hamiltonians.

In contrast, a self-adjoint Hamiltonian on a Hilbert space $\mathcal{I C}$ always has nontrivial integrals (assuming its spectral multiplicity is greater than one). Indeed, this is an obvious consequence of the spectral theorem. Therefore, no abstract analog of Liouville integrability exists for general quantum Hamiltonians.

However, starting from a concrete Liouville integrable system $H(q, \theta), S_{1}(q, \theta), \ldots, S_{N}(q, \theta)$, the question whether a quantization of these Hamiltonians exists such that they still commute is a welldefined one. (Here and below, 'quantization' will mean the substitution

$$
\theta \rightarrow \hat{\theta} \equiv-i \hbar \nabla_{q}
$$

where $\hbar$ denotes Planck's constant.) Whenever the question has an affirmative answer, we shall refer to the operators thus obtained as defining a quantum integrable system. In this sense, all of the systems discussed in Chapter 2 turn out to admit integrable quantum versions.

Next, recall that the notion of Liouville integrability is useless for classical Hamiltonians describing systems of repelling particles on the line, it being always satisfied under mild conditions on the forces. Therefore, we singled out the systems studied in Chapter 2 by first introducing the notion of pure soliton system, cf. Section $2 \mathrm{~A}$.

In the same physical context, this notion has a quantum analog. Specifically, let us assume that the dynamics is sufficiently repulsive for the wave operators [10]

$$
\varepsilon_{ \pm}: \mathcal{K}^{ \pm}=L^{2}\left(G^{ \pm}, d^{N} \theta\right) \rightarrow \mathcal{C}=L^{2}\left(G, d^{N} q\right), \quad G^{ \pm}, G \subset \mathbb{R}^{N}
$$

to exist and be isometric from $\mathcal{T}^{ \pm}$onto $\mathcal{T C}_{C}$. Then we shall say that the dynamics gives rise to a pure soliton system when the $S$-operator

$$
S \equiv \varepsilon_{+}^{-1} \varepsilon_{-}
$$

conserves momenta. (This notion is worked out in more detail in [12].) Observe that the requirement is only useful when more than two particles are involved (just as in the classical case). Note also that we are not requiring that a quantum pure soliton system be a quantum integrable system arising from a classical pure soliton system (this would exclude examples that will be discussed in Section 4C). However, the term 'quantum integrable system' will be used for any quantum pure soliton system, whether it has a classical version or not.

As already mentioned, the classical pure soliton systems $H, S_{1}, \ldots, S_{N}$ of Section 2B can be quantized in such a fashion that the commutativity property is preserved. Except for the $\mathrm{VI}_{\mathrm{rel}}$ case, the commuting operators are (formally) self-adjoint on $L^{2}\left(G, d^{N} q\right)$, where $G$ denotes the classical configuration space. Under such circumstances one expects to obtain quantum pure soliton systems as just defined.

To explain why it is plausible that the soliton property survives quantization whenever the classical Hamiltonians admit commuting, self-adjoint quantizations $H, S_{1}, \ldots, S_{N}$, let us first note that the spectral theorem guarantees the existence of an isometry $\mathscr{E}$ that simultaneously diagonalizes the quantum Hamiltonians on an $L^{2}$-space $\hat{\mathcal{H}} \mathrm{C}$ On physical grounds one expects to be able to choose

$$
\hat{\mathscr{X}}=L^{2}\left(\hat{G}, d^{N} \theta\right) \text {, }
$$

where $\hat{G}$ denotes the definition domain of the classical action variables. Also, the kernel of $\varepsilon$ should be a joint eigenfunction $E(q, \theta), q \in G, \theta \in \hat{G}$. Taking interparticle distances to $\infty$, the operators $S_{1}, \ldots, S_{N}$ reduce to the symmetric functions of the operators $\hat{\theta}_{1}, \ldots, \hat{\theta}_{N}$ and $\exp \left(\beta \hat{\theta}_{1}\right), \ldots$, $\exp \left(\beta \hat{\theta}_{N}\right)$ for the nonrelativistic and relativistic systems, resp. Thus, one expects $E(q, \theta)$ to have plane- 
wave asymptotics. A consideration of the long-time asymptotics then leads to the expectation that the momenta are conserved. In a similar vein, one can argue that the spatial asymptotics of $E(q, \theta)$ should factorize into two-particle quantities, and hence the $S$-operator should factorize too, as a quantum analog of the factorized scattering described in Subsection 2B4. (For more details on these heuristics, see [19].)

Let us now sketch the contents of Section 3B in relation to the above notions. We first detail how the pure soliton systems of Section $2 \mathrm{~B}$ can be quantized in such a fashion that commutativity is preserved. Then we shall try and describe to what extent the scenario of the previous paragraph has been realized for the resulting quantum integrable systems. Let us mention here that even for the simplest case $I_{n r}$ the scenario has not been completely filled in yet. Thus, it is not yet certain that the quantized pure soliton systems of Section $3 \mathrm{~B}$ are quantum pure soliton systems as defined above. (To be sure, finite-dimensional quantum dynamics do exist for which the soliton property has been rigorously proved. We shall return to this in Section 4 C.)

In Section $3 \mathrm{C}$ we shall be concerned with quantum integrable versions of the systems discussed in Section 2C.

\section{B. QUANTIZED PURE SOLITON SYSTEMS}

3B1. Preserving commutativity. The quantization substitution (3.1) leads to unambiguous, (formally) self-adjoint operators $H$ on the Hilbert space $L^{2}\left(G, d^{N} q\right)$ for the cases $\mathrm{I}_{\mathrm{nr}}, \mathrm{II}_{\mathrm{nr}}$ and $\mathrm{VI}_{\mathrm{nr}}$, cf. (2.18)(2.26). This also holds true for the symmetric functions of the Lax matrices (2.37) and (2.38). Indeed, one obtains sums of terms each of which is a product of commuting operators; then self-adjointness is clear from the fact that the classical functions are real-valued. In [5] it is proved that the operators thus obtained commute. The idea of the proof is to exploit classical results: One need only show that the extra terms (compared to the classical case) arising when partials are pushed through sum to zero, since the remaining expression is already known to vanish.

Let us now turn to the relativistic cases. Here, ordering problems already occur for the dynamics $H$, cf. (2.27). We shall first consider the $\mathrm{II}_{\text {rel }}$ case, cf. (2.29). As can be seen from explicit calculations for $N=3$, various 'obvious' choices for the ordering in $H$ and $S_{1}, \ldots, S_{N}$ (cf. (2.42)) spoil commutativity. In this connection it should be pointed out that no a priori argument is known from which the existence of an ordering choice entailing commutativity would follow. However, for the systems at hand such a choice does exist: For any $\hbar$ the operators

$$
S_{l} \equiv \sum_{\substack{I \subset\{1, \ldots, N\} \\|I|=I}} \prod_{\substack{j \in I \\ k \in I}} f_{-}\left(q_{j}-q_{k}\right) \exp \left(\beta \sum_{j \in I} \hat{\theta}_{j}\right) \prod_{\substack{j \in I \\ k \notin I}} f_{+}\left(q_{j}-q_{k}\right)
$$

commute when one sets

$$
f_{ \pm}^{2}(q)=\operatorname{sh}\left(\frac{1}{2} \mu q \pm z\right) / \operatorname{sh} \frac{1}{2} \mu q, \quad z=\frac{1}{2} i \beta \mu g
$$

This claim can be shown to follow from the functional equations

$$
\sum_{\substack{I \subset\{1, \ldots, N\} \\|I|=k}}\left[\prod_{\substack{i \in I \\ j \notin I}} \frac{\operatorname{sh}\left(x_{i}-x_{j}-\gamma\right) \operatorname{sh}\left(x_{i}-x_{j}+\gamma-\rho\right)}{\operatorname{sh}\left(x_{i}-x_{j}\right) \operatorname{sh}\left(x_{i}-x_{j}-\rho\right)}-(x \rightarrow-x)\right]=0
$$

which hold for any $N>1, k \in\{1, \ldots, N\}, x \in \mathbb{C}^{N}, \gamma, \rho \in \mathbb{C}[2]$.

Classical commutativity can now be obtained as a corollary of quantum commutativity. Indeed, the functional equations (3.7) reduce to the functional equations expressing involutivity when one divides by $\rho$ and takes $\rho$ to $0[1,2]$. Moreover, quantum commutativity for the $\mathrm{II}_{\mathrm{nr}}$ case may also be viewed as a corollary.

To explain the latter statement, we introduce the commuting operators

$$
A_{k}(\beta) \equiv \sum_{l=0}^{k}(-)^{k+l}\left(\begin{array}{l}
N-l \\
N-k
\end{array}\right) S_{l}(\beta), \quad k=1, \ldots, N
$$


We have made the $\beta$-dependence explicit, since we are going to consider the nonrelativistic limit $\beta \rightarrow 0$. (The formulas (2.49), (2.50) should be recalled at this point.) Thus, we expand $A_{k}(\beta)$ as a formal power series

$$
A_{k}(\beta)=\sum_{m=0}^{\infty} A_{k}^{(m)} \beta^{m}, \quad \beta \rightarrow 0
$$

and calculate

$$
\begin{aligned}
& A_{1}^{(0)}=0,\left.\quad A\right|^{(1)}=\sum_{j} \hat{\theta}_{j} \\
& A_{2}^{(0)}=A_{2}^{(1)}=0, A_{2}^{(2)}=\sum_{j<k}\left[\hat{\theta}_{j} \hat{\theta}_{k}-g(g-\hbar) \frac{\mu^{2}}{4 \operatorname{sh}^{2} \frac{1}{2} \mu\left(q_{j}-q_{k}\right)}\right]
\end{aligned}
$$

Here, the replacement of $g^{2}$ by $g(g-\hbar)$ as compared to the classical case (recall (2.21)) is due to contributions arising when the partials in (3.5) act on the potentials.

Next, we set

$$
H_{\mathrm{nr}} \equiv-\frac{1}{2} \hbar^{2} \Delta+\frac{1}{4} g(g-\hbar) \mu^{2} \sum_{j<k} \frac{1}{\operatorname{sh}^{2} \frac{1}{2} \mu\left(q_{j}-q_{k}\right)}
$$

and note that

$$
\frac{1}{2} A_{1}(\beta)^{2}-A_{2}(\beta)=\beta^{2} H_{\mathrm{nr}}+O\left(\beta^{3}\right), \quad \beta \rightarrow 0
$$

Therefore, setting

$$
n_{k} \equiv \min \left\{l \mid A_{k}^{(D)} \neq 0\right\}, \quad k=1, \ldots, N
$$

we may conclude that $H_{\mathrm{nr}}$ commutes with $A_{k}^{\left(n_{k}\right)}$. Noting that

$$
A_{k}^{(k)}=\sum_{i_{1}<\cdots<i_{k}} \hat{\theta}_{i_{1}} \cdots \hat{\theta}_{i_{k}}, \quad g=0,
$$

we infer $n_{k} \leqslant k$. Now assume $n_{k}<k$. Then $A_{k}^{\left(n_{k}\right)}$ is a sum of terms arising when one or more partials act on the coefficients of the Taylor series of $f_{+}$around $\beta=0$. Any such term has period $2 \pi i / \mu$ in the $q_{j}$ and vanishes when the interparticle distances are taken to $\infty$, cf. (3.6). Next, consider the sum $M_{k}$ of all terms $T_{k, i}$ in $A_{k}^{\left(n_{k}\right)}$ that are of maximal degree $d_{k}$ in the $\hat{\theta}_{j}$. Since $H_{\mathrm{nr}}$ commutes with $A_{k}^{\left(n_{k}\right)}$, the sum of all terms of degree $d_{k}+1$ in the commutator $\left[\Delta, M_{k}\right]$ must vanish. It then follows from the proof of Lemma 2.5 in a paper by Berezin [30] that the coefficients of the $T_{k, i}$ are polynomials in the $q_{j}$. By periodicity these coefficients must then be constants, and taking interparticle distances to $\infty$, it follows that these constants are zero. Hence we infer $A_{k}^{\left(n_{k}\right)}=0$, which contradicts (3.14). Therefore, we must have $n_{k}=k$.

The upshot is, that we have now proved

$$
\begin{array}{ll}
{\left[H_{\mathrm{nr}}, A_{k}^{(k)}\right]=0,} & k=1, \ldots, N \\
{\left[A_{k}^{(k)}, A_{l}^{(l)}\right]=0,} & k, l=1, \ldots, N
\end{array}
$$

However, the above arguments do not yield an important piece of information on $A_{k}^{(k)}$ : it must be the $k^{\text {th }}$ symmetric function of the quantized Lax matrix $(2.37)$, with $g$ replaced by $[g(g-\hbar)]^{1 / 2}$. Indeed, as already mentioned, the latter operator commutes with $H_{\mathrm{nr}}$ [5]. Subtracting $A_{k}^{(k)}$ yields an operator commuting with $H_{\mathrm{nr}}$ that must be zero by the above reasoning: The point is again that the difference consists of $(2 \pi i / \mu)$-periodic terms that vanish for $\left|q_{j}-q_{k}\right| \rightarrow \infty$.

Thus far we have restricted ourselves to the systems of type II. The relevant information for the type I systems now easily follows from the above by taking $\mu$ to 0 . We proceed by considering the 
quantization of the $\mathrm{VI}_{\text {rel }}$ functions (2.48). Here, the ordering choice that 'works' is given by

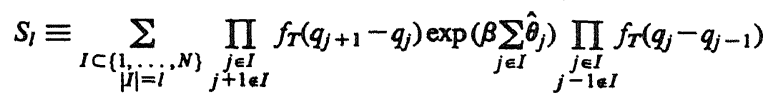

This can be proved either directly [3] or by reduction to the $\mathrm{I}_{\text {rel }}$ case via the substitutions (2.24), (2.35) and the limit $\epsilon \rightarrow 0$. In the same way as for the $\mathrm{II}_{\text {rel }}$ case, $\mathrm{VI}_{\text {rel }}$ quantum commutativity now leads to commutativity for the classical $\mathrm{VI}_{\mathrm{rel}}$ and quantum $\mathrm{VI}_{\mathrm{nr}}$ systems.

The $\mathrm{II}_{\text {rel }}$ operators (3.5) are (formally) self-adjoint, provided $z \in i \mathbb{R}$. In contrast, the $\mathrm{VI}_{\text {rel }}$ operators (3.18) are not self-adjoint (except when $\hbar \beta \mu=2 \pi k, k \in \mathbb{Z}$ ). When one takes $z=\gamma+i \pi / 2$ with $\gamma \in \mathbb{R}^{*}$ in (3.5), self-adjointness is also violated. (Recall the $\mathrm{VI}_{\text {rel }}$ case arises for $\gamma \rightarrow \infty$.) Thus, if a joint eigenfunction transform exists for the latter cases, it is not going to be isometric.

From now on we use the convention $\hbar \equiv 1$. Since $g$ has the dimension of action, it should cause no surprise that the above operators turn out to have special properties for $g \in \mathbb{N}$. We note in particular that (3.5), (3.6) entail

$$
S_{k}=\sum_{1<i_{1}<\cdots<i_{k}<N} \exp \left[\beta\left(\hat{\theta}_{i_{1}}+\cdots+\hat{\theta}_{i_{k}}\right)\right], \quad g=1
$$

Hence, the quantum $\mathrm{II}_{\mathrm{re}}$ systems are free for $z=\frac{1}{2} i \beta \mu$.

3B2. The eigenfunction transform and duality. For special values of $g$ (in particular $1 / 2,1$ and 2) the commuting $\mathrm{I}_{\mathrm{nr}} / \mathrm{II}_{\mathrm{nr}}$ operators can be related to the radial parts of the Laplace operators on zero/negative curvature Riemannian symmetric spaces with restricted root system $A_{N-1}$. (More precisely, this is the case when the center-of-mass coordinate is omitted.) The $\mathrm{VI}_{\mathrm{nr}}$ systems can also be tied in with harmonic analysis on symmetric spaces.

For the cases just mentioned the eigenfunctions are known explicitly and have been studied in great detail. (A lucid and nontechnical survey of results obtained in the symmetric space context can be found in [5]; systematic and detailed treatments are presented in [31-33].) In particular, for each permutation $\sigma$ there exists a unique function $E_{\sigma}$ satisfying

$$
\begin{aligned}
& S_{k} E_{\sigma}(q, \theta)=E_{\sigma}(q, \theta) \sum_{i_{1}<\cdots<i_{k}} \theta_{i_{1}} \cdots \theta_{i_{k}}, \quad k=1, \ldots, N \\
& E_{\sigma}(q, \theta) \sim \exp \left(i q \cdot \theta_{\sigma}\right), \quad q_{N} \ll<q_{N-1} \cdots \ll<q_{1}
\end{aligned}
$$

where

$$
\theta_{\sigma} \equiv\left(\theta_{\alpha(1)}, \ldots, \theta_{\alpha(N)}\right)
$$

Moreover, a suitable linear combination $E(q, \theta)$ of these $N$ ! joint eigenfunctions solves the Plancherel problem. That is, the operator

$$
\text { E: } \hat{\mathcal{C}} \equiv L^{2}\left(\hat{G}, d^{N} \theta\right) \rightarrow \mathcal{X} \equiv L^{2}\left(G, d^{N} q\right), f \mapsto \int_{G} d^{N} \theta E(\cdot, \theta) f(\theta)
$$

is an isometry from $\hat{\mathscr{C}}$ onto $\mathscr{S}$, where

$$
\hat{G} \equiv\left\{y \in \mathbf{R}^{N} \mid y_{N}<\cdots<y_{1}\right\}, \quad G \equiv \begin{cases}\hat{G}, & \left(\mathrm{I}_{\mathrm{nr}}, \mathrm{II}_{\mathrm{nr}}\right) \\ \mathbf{R}^{N}, & \left(\mathrm{VI}_{\mathrm{nr}}\right)\end{cases}
$$

(cf. (2.18), (2.19), (2.51)). Of course, the case $g=1$ is trivial: Then (3.12) corresponds to the radial part of the Laplace-Beltrami operator on $G L(N, C) / S U(N)$, and one obtains

$$
E_{o}(q, \theta)=\exp \left(i q \cdot \theta_{0}\right), \quad E=(2 \pi)^{-N / 2} \sum_{\sigma}(-)^{\sigma} E_{0}, \quad g=1
$$


Recently, joint eigenfunctions for the $\mathrm{II}_{\mathrm{nr}}$ case with $\mathrm{g}$ taking arbitrary values have been constructed by Heckman and Opdam; moreover, their results apply to arbitrary root systems [34-37]. The above picture for the symmetric space values of $g$ is not substantially modified, but the Plancherel formula ('orthogonality and completeness') has not been proven yet.

Let us now turn to the relativistic cases $\mathrm{I}_{\text {rel }}, \mathrm{II}_{\text {rel }}$ and $\mathrm{VI}_{\text {rel }}$. Here, the quest for explicit joint eigenfunctions leads to problems of a novel nature, most of which have not been solved to date.

To explain some of the difficulties involved, and to sketch some of our results obtained thus far, we specialize to the $\mathrm{I}_{\text {rel }}$ and $\mathrm{II}_{\text {rel }}$ cases with $N=2$. (Even for $N=2$ we have no information on eigenfunctions for the $\mathrm{VI}_{\text {rel }}$ case.) Then the problem is to find eigenfunctions for the operator

$$
H=\left(\frac{\operatorname{sh} v(q-i \beta g)}{\operatorname{sh} v q}\right)^{\frac{1}{2}} T_{i \beta}\left(\frac{\operatorname{sh} v(q+i \beta g)}{\operatorname{sh} v q}\right)^{\frac{1}{2}}+(\beta \rightarrow-\beta) .
$$

Here, we have introduced the formal translation operator

$$
\left(T_{\xi} f\right)(q) \equiv f(q-\xi), \quad \quad \xi \in \mathbb{C}
$$

and the new parameter

$$
\nu \equiv \mu / 2 \text {. }
$$

(When we would stick to $\mu$, various factors 2 would arise due to our use of asymmetric center-of-mass variables

$$
q \equiv \dot{q}_{1}-q_{2}, \quad \theta \equiv\left(\theta_{1}-\theta_{2}\right) / 2,
$$

cf. also (3.5), (3.6) with $N=2, l=1, \hbar=1$.) More in detail, for $N=2$ the key question is, whether a function $E$ exists such that

$$
\frac{1}{2} H E(q, \theta)=E(q, \theta) \operatorname{ch} \beta \theta
$$

and such that the operator

$$
(\delta f)(q) \equiv \int_{0}^{\infty} d \theta E(q, \theta) f(\theta), \quad f \in C_{0}^{\infty}((0, \infty))
$$

gives rise to an isometry between the Hilbert spaces

$$
\hat{\mathcal{Y}} \equiv L^{2}([0, \infty), d \theta), \quad \mathcal{K} \equiv L^{2}([0, \infty), d q) .
$$

There appear to be no results in the vast literature on eigenfunction expansions that have a direct bearing on this question. More generally, the (once very active) study of analytic difference equations $(A \Delta E S)$ and analytic difference operators $(A \Delta O s)$ (such as (3.30) and (3.26), resp.) seems to have been abandoned, by and large, before functional analysis and quantum mechanics really got off the ground. Indeed, the last full-fledged monograph devoted to $A \Delta E s$ appears to date from 1924 [38]. (More recent studies do exist, cf. e.g. [39-42]; however, just as in [38], the questions dealt with are of a quite different character.)

To explain the key difference between a second-order ODE or discrete difference equation and the second-order $A \Delta E$ (3.30), we recall that the solution space is two-dimensional in the former context, and that the Plancherel problem is solved by the Weyl-Kodaira-Titchmarsh theory. In contrast, for the $A \Delta E$ (3.30) even the existence of any solution is a priori unclear. However, this problem can be solved by invoking the extensive lore gathered mostly in the $19^{\text {th }}$ century. Specifically, solutions can be constructed by recursive procedures [38]. Unfortunately, such solutions are often badly singular; even natural boundaries can easily occur. But the existence of even one non-trivial solution $E(q, \theta)$ entails the existence of an infinite-dimensional solution space. Indeed, for any $F(q, \theta)$ with period $i \beta$ in $q$ the function $F(q, \theta) E(q, \theta)$ is another solution. As a more restrictive fact, we may mention that 
the solution space is a two-dimensional vector space over the field of $i \beta$-periodic functions [38], but all of these results fall far short of settling the key question mentioned above.

A closely related question is: How can one turn the $A \Delta O$ (3.26) into a self-adjoint operator on $M C$ ? Again, we have no general answers to offer. Recall in this connection that the operator $-i d / d q$ on the dense domain $C_{0}^{\infty}((0, \infty)) \subset \mathcal{C H}$ is the standard example of a symmetric operator without self-adjoint extensions. Thus, even for the simple $A \Delta O$

$$
T_{i \beta}=\exp (-i \beta d / d q) \quad \beta \in(0, \infty)
$$

an interpretation as a self-adjoint operator on $\mathcal{T}$ is problematic.

Having provided some context, we are now prepared to present some explicit answers to the above question for the $\mathrm{I}_{\text {rel }}$ and $\mathrm{II}_{\mathrm{rel}}$ cases. First of all, it so happens that the answer for the $N=2 \mathrm{I}_{\text {rel }}$ case (obtained by taking $\nu \rightarrow 0$ in (3.26)) can already be found in the literature (once one realizes where to look). To substantiate this assertion, we invoke the (known) eigenfunction transform for the $\mathrm{II}_{\mathrm{ar}}$ Hamiltonian

$$
\hat{H} \equiv-\frac{d^{2}}{d \theta^{2}}+\beta^{2} g(g-1) \frac{1}{\operatorname{sh}^{2} \beta \theta}, \quad g \geqslant \frac{1}{2}
$$

whose kernel reads

$$
\begin{gathered}
\left.E(\beta, g ; q, \theta)=2^{-g+\frac{1}{2}} \Gamma\left(g+\frac{1}{2}\right)^{-1}(\operatorname{sh} \beta \theta)\right)^{g}\left[\frac{\Gamma(g-i q / \beta) \Gamma(g+i q / \beta)}{\Gamma(-i q / \beta) \Gamma(i q / \beta)}\right]^{\frac{1}{2}} \\
{ }_{2} F_{1}\left(\frac{1}{2}(g+i q / \beta), \frac{1}{2}(g-i q / \beta), g+\frac{1}{2} ;-\operatorname{sh}^{2} \beta \theta\right), \quad q, \theta>0 .
\end{gathered}
$$

The crux is, that the contiguous relations for the hypergeometric function and the $A \Delta E$ $\Gamma(1+z)=z \Gamma(z)$ entail that the function (3.35) satisfies the $A \Delta E(3.30)$. Moreover, for $g \geqslant 1 / 2$ the operator $\mathcal{E}$ defined by (3.31) extends to an isometry from $\hat{\mathcal{C}}$ onto $\mathcal{X}$ by virtue of the Weyl-KodairaTitchmarsh theory. Therefore, the above key question has an affirmative and explicit answer for the $N=2 \mathrm{I}_{\text {rel }}$ case.

We conjecture that the $N>2$ existence question for the $\mathrm{I}_{\text {rel }}$ regime has already been answered in the literature too. Specifically, we expect that the $\mathrm{II}_{\mathrm{nr}}$ eigenfunctions of Heckman and Opdam [34,35] are joint eigenfunctions for the commuting $\mathrm{I}_{\text {rel }}$ operators, acting on the spectral variables. More in detail, this should hold after an obvious similarity transformation turning the Lebesgue measure used here into the Plancherel measure used in harmonic analysis, and after a suitable normalization of the dependence on the spectral variables. (Also, we should repeat at this point that the Plancherel problem has only been solved for the group values of $g$.)

Of course, what is being said here, is that we expect the duality properties of the classical level to survive quantization, cf. (2.53). For the arbitrary $N \mathrm{I}_{\mathrm{nr}}$ case this conjecture can easily be verified for the group values of $g$ : It amounts to the fact that $E(q, \theta)=E(\theta, q)$. Furthermore, for $N=2$ this selfduality property is evident without restriction on $g$. Indeed, then the desired isometry is in essence the Hankel transform, whose kernel depends only on the product $q \theta$.

Similarly, we expect the $\mathrm{II}_{\mathrm{rel}}$ transform to satisfy

$$
E(\beta, v, g ; q, \theta)=E(\nu, \beta, g ; \theta, q) \text {. }
$$

This holds true for all cases where we have found explicit solutions. In particular, it holds when

$$
g=M+1=1,2,3, \cdots
$$

For these $g$-values the following functions solve the $A \Delta E$ (3.30) and have the self-duality property (3.36):

$F_{M}(q, \theta) \equiv|A(v q) A(\beta \theta)| \exp (i q \theta-M v q-M \beta \theta) \sum_{k, l=0}^{M}(-)^{k+l} Q_{k l} \exp (2 k v q+2 / \beta \theta)$ 
Here, the function $A(x)$ and the matrix $Q_{k l}$ depend on $v$ and $\beta$ only via their product

$$
\alpha \equiv \beta v
$$

Hence, self-duality amounts to $Q_{k l}$ being symmetric. Explicitly, one has

$$
\begin{gathered}
A(x) \equiv \prod_{j=1}^{M}[2 \operatorname{sh}(x+i j \alpha)]^{-1} \\
Q_{k l} \equiv \exp (i \alpha M(M+1) / 2) \sum_{1<i_{1}<\cdots<i_{k}<M} \exp \left(-2 i \alpha\left[i_{1}+\cdots+i_{k}\right]\right) \\
\sum_{\substack{-M<j_{1}<\cdots<j_{l}<M \\
j_{\star} \notin\{-M+k, \ldots,-1+k, k\}}} \exp \left(-2 i \alpha\left[j_{1}+\cdots+j_{l}\right]\right)
\end{gathered}
$$

(Here, empty sums equal 1 by definition, so that $F_{0}(q, \theta)=\exp (i q \theta)$, e.g.) We have not found a manifestly symmetric formula for $Q_{k l}$. However, it is easy to check symmetry for small $M$, and we shall prove symmetry for arbitrary $M$ elsewhere, as well as the claim that $F_{M}(q, \theta)$ solves the $A \Delta E(3.30)$.

We shall also omit the proofs of the assertions that now follow [43]. First, not only $F_{M}$, but also $\bar{F}_{M}$ solves (3.30). Second, the real-valued kernel

$$
E_{M}(q, \theta) \equiv(2 \pi)^{-1 / 2}(-i)^{M+1}\left[F_{M}(q, \theta)-(-)^{M} \bar{F}_{M}(q, \theta)\right], \quad M \in \mathbb{N}
$$

defines an isometry $\varepsilon_{M}$ from $\hat{\mathcal{C}}$ onto $\mathcal{X}$ for any $\alpha \in(0, \pi / M]$, thus solving the Plancherel problem posed above, cf. (3.30)-(3.32). Third, the operator

$$
\left(\digamma_{M} f\right)(q) \equiv(2 \pi)^{-1 / 2}(-i)^{M+1} \int_{-\infty}^{\infty} d \theta F_{M}(q, \theta) f(\theta), \quad f \in L^{2}(\mathbf{R}), \quad M \in \mathbb{N}^{*}
$$

preserves parity and amounts to $\mathscr{E}_{M}$ on the subspace $L^{2}(\boldsymbol{R})_{a}$ of odd functions. However, $\mathscr{F}_{M}$ is not isometric on $L^{2}(\mathbb{R})_{s}$, even though the even kernel has properties similar to the odd one: It has in essence the same plane-wave asymptotics for $|q| \rightarrow \infty$, is real-analytic on $\mathbb{R}$, and solves the self-adjoint $A \Delta E$ (3.30). (In fact, its deviation from being an isometry can be explicitly described.)

3B3. Soliton scattering. As mentioned in the previous subsection, a linear combination $E(q, \theta)$ of the functions (3.21) solves the Plancherel problem for the group values of $g$. Specifically, one may take

$$
E(q, \theta)=(2 \pi)^{-N / 2} \sum_{\sigma \in S_{N}}(-)^{\sigma} \prod_{\substack{i<j-1 \\ \sigma^{-1}(i)<\sigma^{-1}(j)}} u\left(\frac{1}{2}\left(\theta_{i}-\theta_{j}\right)\right)^{1 / 2} \prod_{\substack{i<j \\ \sigma^{-1}(i)>\sigma^{-1}(j)}} u\left(\frac{1}{2}\left(\theta_{i}-\theta_{j}\right)\right)^{-1 / 2} E_{\sigma}(q, \theta)
$$

where $u(\theta)$ is the two-particle $S$-matrix,

$$
u(\theta)= \begin{cases}\exp [i \pi(1-g)] & \left(\mathrm{I}_{\mathrm{nr}}\right) \\ \frac{\Gamma(1+2 i \theta / \mu) \Gamma(g-2 i \theta / \mu)}{\Gamma(1-2 i \theta / \mu) \Gamma(g+2 i \theta / \mu)} & \left(\mathrm{II}_{\mathrm{nr}}\right) \\ -\frac{\Gamma(2 i \theta / \mu)}{\Gamma(-2 i \theta / \mu)} \exp [4 i(\ln \mu) \theta / \mu] & \left(\mathrm{VI}_{\mathrm{nr}}\right)\end{cases}
$$

More generally, for any $g \geqslant 1 / 2$ the Heckman/Opdam function with the same structure is the obvious candidate for an isometry from $\hat{\mathcal{C}}$ onto $\mathcal{X}$. Reading off the $S$-matrix from (3.44) according to the standard recipe of formal scattering theory, one finds that $S$ is the unitary multiplication operator

$$
S(\theta)=\prod_{1<i<j<N} u\left(\frac{1}{2}\left(\theta_{i}-\theta_{j}\right)\right)
$$

To show that the $I_{n r}, I_{n r}$ and $\mathrm{VI}_{\mathrm{nr}}$ systems are quantum pure soliton systems as defined in Section 
$3 \mathrm{~A}$, one would have to prove that the wave operators $\mathscr{E}_{ \pm}$exist, that they have range $\mathscr{K}$, and that $S=\mathcal{E}_{+}^{-1} \mathcal{E}_{-}$is given by (3.46). We expect that this is true for any $g \geqslant 1 / 2$, the kernels of $\mathcal{E}_{ \pm}$being given by the incoming and outgoing eigenfunctions

$$
E_{ \pm}(q, \theta)=E(q, \theta) S(\theta)^{\mp / 2}
$$

when one takes as comparison operator the generalized sine transform (3.25).

Let us now turn to the relativistic cases $\mathrm{I}_{\text {rel }}$ and $\mathrm{II}_{\text {rel }}$, taking $N \equiv 2$ from now on. Using the known asymptotics of the $\Gamma$-function and the hypergeometric function, one obtains from (3.35)

$$
u(\theta)=\exp [i \pi(1-g)] \quad\left(I_{\mathrm{rel}}\right)
$$

Continuing with the $\mathrm{II}_{\text {rel }}$ case, we obtain

$$
u(\theta)=(-)^{M} \prod_{k=1}^{M} \frac{\operatorname{sh}(\beta \theta+i k \alpha)}{\operatorname{sh}(\beta \theta-i k \alpha)}, \quad g=M+1 \quad\left(I_{\mathrm{rel}}\right)
$$

from the explicit transforms (3.38)-(3.42).

We can also determine $u$ for arbitrary g, provided some assumptions are made. Suppose $E(q, \theta)$ is a solution to (3.30) with asymptotics

$$
E(q, \theta) \sim(2 \pi)^{-1 / 2}\left[u(\theta)^{1 / 2} \exp (i q \theta)-u(\theta)^{-1 / 2} \exp (-i q \theta)\right], \quad q \rightarrow \infty
$$

If we assume in addition that $E$ is self-dual (i.e., that (3.36) holds true), then it follows that $E$ satisfies an $A \Delta E$ in $\theta$, too. Specifically, one must have

$$
\frac{1}{2} \hat{H} E(q, \theta)=\operatorname{chv} q E(q, \theta)
$$

where $\hat{H}$ is the $A \Delta O$ dual to (3.26),

$$
\begin{aligned}
& \hat{H}=f_{-}(\theta) \hat{T}_{i v} f_{+}(\theta)+f_{+}(\theta) \hat{T}_{-i v} f_{-}(\theta) \\
& f_{ \pm}(\theta) \equiv\left[\frac{\operatorname{sh} \beta(\theta \pm i v g)}{\operatorname{sh} \beta \theta}\right]^{\frac{1}{2}}
\end{aligned}
$$

Let us now consider (3.51) for $q \rightarrow \infty$, using the asymptotics (3.50). Comparing leading terms, it follows that $u(\theta)$ satisfies the $A \Delta E$

$$
\frac{u\left(\theta+\frac{1}{2} i v\right)}{u\left(\theta-\frac{1}{2} i v\right)}=F(\theta)
$$

where

$$
F(\theta)=f_{+}^{2}\left(\theta-\frac{1}{2} i v\right) f_{-}^{2}\left(\theta+\frac{1}{2} i v\right)
$$

The point of all this is, that first-order $A \Delta E$ of the form (3.54) can be solved explicitly for large classes of right-hand sides. Moreover, the solution is unique when certain analyticity requirements are imposed. In particular, for the special case just encountered, we find [44] that the solution satisfying $u(0)=1$ is given by

$$
u(\theta)=\exp \left[2 i \int_{0}^{\infty} \frac{d x}{x} \frac{\operatorname{sh}(\alpha-\tau) x}{\operatorname{sh} \alpha x} \frac{\operatorname{sh}(\pi-\tau) x}{\operatorname{sh} \pi x} \sin 2 \beta x \theta\right]
$$

provided the parameters

$$
\alpha \equiv \beta v=\frac{1}{2} \beta \mu, \quad \tau \equiv \alpha g=-i z
$$


belong to

$$
R \equiv\left\{(\alpha, \tau) \in \mathbb{R}^{2} \mid \alpha \in(0,2 \pi], \tau-\frac{1}{2} \alpha \in[0, \pi]\right\}
$$

The $S$-matrix (3.56) has various remarkable properties that do not meet the eye. For instance, it is an elementary function for a dense set $\oslash \subset R$. In particular, one has

$$
u(\theta)=(-)^{M+L} \prod_{k=1}^{M} \frac{\operatorname{sh}(\beta \theta+i k \alpha)}{\operatorname{sh}(\beta \theta-i k \alpha)} \prod_{l=1}^{L} \frac{\operatorname{sh}\left(\pi \theta / v+i l \pi^{2} / \alpha\right)}{\operatorname{sh}\left(\pi \theta / \nu-i l \pi^{2} / \alpha\right)}
$$

for points in the set

$$
\Phi_{1} \equiv\{(\alpha, \tau) \in R \mid \tau \equiv(M+1) \alpha-L \pi, M \geqslant 1, L \geqslant 0\}
$$

which is already dense. For $(\alpha, \tau) \in \mathscr{D} \supset \mathscr{D}_{1}$ there exist elementary self-dual solutions to the $A \Delta E(3.30)$ with the asymptotics (3.50), (3.56). However, the corresponding transforms are not isometric, in general. To date, we have not been able to prove or disprove the existence of self-dual periodic multipliers that correct this. On the other hand, a natural generalization of the Harish-Chandra $c$-function does exist for any $(\alpha, \tau) \in R[44]$.

\section{C. SYSTEMS RELATED TO QUANTIZED PURE SOLTTON SYSTEMS}

3C1. Systems with solitons and antisolitons. The operators associated with the $\tilde{\mathrm{II}}_{\mathrm{nr}}$ and $\tilde{\mathrm{I}}_{\mathrm{rel}}$ cases are obtained from the commuting operators of the $\mathrm{II}_{\mathrm{nr}}$ and $\mathrm{II}_{\mathrm{rel}}$ cases via the substitution (2.78). Since this amounts to an analytic continuation, it is obvious that the former operators also commute (as formal PDOs and $A \Delta O$ s, resp.). For special values of $g$ the commuting $\tilde{\mathrm{I}}_{\mathrm{nr}}$ operators can be tied in with harmonic analysis on pseudo-Riemannian symmetric spaces. However, even in these special cases much less is known concerning the joint eigenfunctions than for the $\mathrm{II}_{\mathrm{nr}}$ case. In particular, one is still far removed from a verification of the picture of factorized scattering pertaining to the $\tilde{I}_{\mathrm{nr}}$ systems. This picture is taken for granted in the physics literature (cf. e.g. [45]), and will now be summarized.

First, recall we have already discussed the corresponding classical situation in Subsection $2 \mathrm{Cl}$. The multi-channel scattering described there is believed to persist at the quantum level (for $g>1$ ). However, in any channel with asymptotically free solitons and antisolitons (from now on $s$ and $\bar{s}$, resp.) there occurs a key difference with the classical case: an $\bar{s}$ collison leads to a non-zero reflection for $g \notin N$. Because it is still assumed that a multi-body scattering amplitude can be written as a product of 2-body amplitudes corresponding to an arbitrarily chosen temporal order for the 2-body collisons involved, the product must be independent of this choice. Consequently, one obtains constraint equations for the $s s$ and $\bar{s} \bar{s}$ reflection coefficient $u$ and the $\bar{s} \bar{s}$ transmission and reflection coefficients $t$ and $r$. These cubic equations are the well-known Yang-Baxter equations, and they are indeed satisfied for the functions $u, t$ and $r$ of the $N=2$ case. This is a consequence of the relations

$$
\begin{aligned}
& t(\theta)=\frac{\operatorname{sh}(\pi \theta / v)}{\operatorname{sh}(i \pi g-\pi \theta / v)} \quad u(\theta), \quad \theta>0 \\
& r(\theta)=\frac{\operatorname{sh}(i \pi g)}{\operatorname{sh}(i \pi g-\pi \theta / v)} \quad u(\theta), \quad \theta>0
\end{aligned}
$$

which can be verified from the $\overline{s s}$ eigenfunction transform. (The latter is obtained by taking a suitable linear combination of $E(v, g ; \theta, q \pm i \pi / 2 y)$, cf. (3.35).)

Consider now, more generally, the $\tilde{\mathrm{I}}_{\mathrm{rel}}$ case. We expect that the picture just sketched for the $\tilde{\mathrm{I}}_{\mathrm{nr}}$ case applies here, too, with $t, r$ and $u$ still related via (3.61) and (3.62). In particular, for the solutions detailed in Subsection 3B2 we have $g \in \mathbf{N}$, so that (3.62) says $r=0$. This is indeed the case for the $\bar{s}$ eigenfunction transforms following from (3.38)-(3.41).

The physical fact that the interaction between $s$ and $\bar{s}$ is attractive for $g>1$ finds its mathematical expression in the occurrence of bound states. For the $\tilde{\mathrm{I}}_{\mathrm{nr}}$ operator 


$$
H_{s \bar{s}}=-\frac{d^{2}}{d q^{2}}-v^{2} g(g-1) / \mathrm{ch}^{2} v q
$$

these have energies

$$
E_{n}=-v^{2}[g-(n+1)]^{2}, \quad n=0, \ldots, M-1, \quad g \in(M, M+1]
$$

and the corresponding wave functions read

$$
\psi_{n}(q)=P_{n}(i \operatorname{sh} v q) \psi_{0}(q), \quad n=0, \ldots, M-1, \quad g \in(M, M+1]
$$

Here, $P_{n}$ are Gegenbauer polynomials, and the ground state may be taken to be

$$
\psi_{0}(q)=[2 \operatorname{ch} v q]^{1-g}
$$

Since one has

$$
\psi_{0}(q) \sim \exp [v|q|(1-g)], \quad|q| \rightarrow \infty
$$

the functions $\psi_{n}(q)$ are indeed in $L^{2}(\mathbb{R})$.

For the $\tilde{I}_{\text {rel }}$ Hamiltonian

$$
H_{s \bar{s}}=\left(\frac{\operatorname{ch} v(q-i \beta g)}{\operatorname{ch} v q}\right)^{\frac{1}{2}} T_{i \beta}\left(\frac{\operatorname{ch} v(q+i \beta g)}{\operatorname{ch} v q}\right)^{\frac{1}{2}}+(\beta \rightarrow-\beta)
$$

the odd and even eigenfunction transforms can be found explicitly for $g=M+1 \in \mathbb{N}$ via analytic continuation of the solutions (3.38), and their isometry properties are known [43]. There are $M$ bound states and the ground state wave function can be taken to be

$$
\psi_{0}(q)=\prod_{j=1}^{M}|2 \operatorname{ch}(v q+i j \alpha)|^{-1}
$$

More generally, explicit square-integrable and pairwise orthogonal eigenfunctions of the formal $A \Delta O$ (3.68) can be found for $g>1$. The ground state, normalized to satisfy (3.67), reads [44]

$$
\psi_{0}(q)=\exp \left[(\alpha-\tau) / \pi+\int_{0}^{\infty} d x\left[\frac{\operatorname{sh}(\tau-\alpha) x \operatorname{ch} \tau x \cos 2 x v q}{x \operatorname{sh} \pi x \operatorname{sh} \alpha x}-\frac{\alpha(\tau-\alpha)}{\pi \operatorname{sh}^{2} \alpha x}\right]\right]
$$

and then the excited states are given by (3.65), where the $P_{n}(x)$ are again polynomials of degree $n$ and parity $(-)^{n}$. (These polynomials are in essence $q$-Gegenbauer polynomials, cf. Subsection $3 \mathrm{C} 2$ below.) As the generalization of (3.64), we obtain the eigenvalues

$$
E_{n}=2 \cos (\tau-(n+1) \alpha), \quad n=0, \ldots, M-1, \quad g \in(M, M+1], \quad \tau=\alpha g<\alpha+\frac{\pi}{2}
$$

The function (3.70) is an elementary function for a dense set in a region obtained by shifting (3.58). In particular, when $\tau=\pi / 2(3.70)$ can be written

$$
\psi_{0}(q)=(\operatorname{sh} 2 v q / \operatorname{sh} \pi q / \beta)^{\frac{1}{2}} \quad \tau=\pi / 2
$$

and when $\tau=(M+1) \alpha(3.70)$ reduces to (3.69) [44].

3C2. Sustherland type systems and their duals. Just as for the $\tilde{\mathrm{I}}_{\mathrm{nr}}$ and $\tilde{\mathrm{I}}_{\mathrm{rel}}$ cases, commutativity of the quantized Hamiltonians associated with the regimes $\mathrm{III}_{\mathrm{nr}}, \mathrm{III}_{\mathrm{rel}}$ and $\mathrm{III}_{\mathrm{rel}, b}$ is clear from the fact that these operators can be obtained by analytic continuation from the $\mathrm{II}_{\mathrm{nr}}$ and $\mathrm{II}_{\mathrm{rel}}$ regimes, resp. We continue by sketching the state of the art as concerns their joint eigenfunctions. To this end it is expedient to discuss the $N=2$ case first.

Omitting the center-of-mass motion, the relevant operators and their duals read

$$
H=-\frac{d^{2}}{d q^{2}}+v^{2} g(g-1) / \sin ^{2} v q
$$




$$
\begin{aligned}
& \hat{H}=\left[1+\frac{v g}{\theta}\right)^{\frac{1}{2}} \hat{T}_{-v}\left(1-\frac{v g}{\theta}\right)^{\frac{1}{2}}+(v \rightarrow-v) \quad \text { (III } \\
& H=\left(\frac{\sin v(q-i \beta g)}{\sin v q}\right)^{\frac{1}{2}} T_{i \beta}\left(\frac{\sin v(q+i \beta g)}{\sin v q}\right)^{\frac{1}{2}}+(\beta \rightarrow-\beta) \quad\left(\mathrm{II}_{\mathrm{rel}}\right) \\
& \hat{H}=\left(\frac{\operatorname{sh} \beta(\theta+v g)}{\operatorname{sh} \beta \theta}\right)^{\frac{1}{2}} \hat{T}_{-v}\left(\frac{\operatorname{sh} \beta(\theta-v g)}{\operatorname{sh} \beta \theta}\right)^{\frac{1}{2}}+(v \rightarrow-v) \quad\left(\hat{I I I ~}_{\mathrm{red}}\right) \\
& H=\left(\frac{\sin v(q+\beta g)}{\sin v q}\right)^{\frac{1}{2}} T_{-\beta}\left(\frac{\sin v(q-\beta g)}{\sin v q}\right)^{\frac{1}{2}}+(\beta \rightarrow-\beta) \quad\left(\mathrm{III}_{\mathrm{rel}, b}\right) \\
& \left.\hat{H}=\left(\frac{\sin \beta(\theta+v g)}{\sin \beta \theta}\right)^{\frac{1}{2}} \hat{T}_{-v}\left(\frac{\sin \beta(\theta-v g)}{\sin \beta \theta}\right)^{\frac{1}{2}}+(v \rightarrow-v) \quad \text { (III }{ }_{\text {rel, }, b}\right)
\end{aligned}
$$

Here, we are taking $\beta, v \in(0, \infty)$ in all cases, in contrast to Subsection 2C2. The change $g^{2} \rightarrow g(g-1)$ in (3.73) (as compared to Subsection 2C2) is a natural consequence of the $\mathrm{III}_{\mathrm{rel}} \rightarrow \mathrm{II}_{\text {nr }}$ transition at the quantum level, cf. Subsection 3B1. Recall also that $T_{\xi}$ and $\hat{T}_{\xi}$ denote translation of $q$ and $\theta$, resp., cf. (3.27).

For $g \geqslant 3 / 2$ the operator (3.73) is essentially self-adjoint on $C_{0}^{\infty}((0, \pi / v)) \subset S C$, where

$$
\mathcal{C} \equiv L^{2}([0, \pi / v], d q)
$$

Its closure has purely discrete spectrum $\left\{v^{2}(g+n)^{2} \mid n \in \mathbf{N}\right\}$ and a corresponding basis for $\mathcal{K}$ can be taken to be

$$
\psi_{n}(q)=P_{n}(\cos v q) \psi_{0}(q)
$$

where the $P_{n}$ are Gegenbauer polynomials with weight function

$$
\psi_{0}(q)^{2}=(\sin v q)^{28}
$$

Correspondingly, the dual space may be taken to be

$$
\hat{\mathscr{C}} \equiv \boldsymbol{l}^{2}(\hat{G}), \quad \hat{G} \equiv\{v g+v n \mid n \in \mathbb{N}\}
$$

and the duality of the classical level is preserved. Indeed, when one discretizes the $\theta$-variable in (3.74) by taking $\theta \in G$, then the function

$$
E\left(q, \theta_{n}\right) \equiv \psi_{n}(q), \quad \theta_{n} \equiv v g+v n
$$

is an eigenfunction of $\hat{H}$ with eigenvalue $2 \cos v q$ by virtue of the three-term recurrence relation of the (suitably normalized) Gegenbauer polynomials.

For the $\mathrm{III}_{\mathrm{rd}}$ regime this state of affairs persists, in essence. The eigenfunctions are again of the form (3.80); the weight function (3.81) and its associated orthogonal polynomials should be replaced by certain $q$-analogs. Specifically, the $q$-Gegenbauer polynomials studied in $[46,47]$ arise in this way. (This was pointed out to the author by T. Koornwinder [48].) The parameters employed by Askey and Ismail [46] are related to ours by

$$
q_{A I}=\exp (-2 \beta v), \quad \beta_{A I}=\exp (-2 \beta v g), \quad \lambda_{A I}=g
$$

Hence, the limit $q_{A I} \rightarrow 1$ may be viewed as the nonrelativistic limit $c \rightarrow \infty$. Again, the duality of the classical level survives quantization: The three-term recurrence relation for the (appropriately normalized) $q$-Gegenbauer polynomials implies that the generalization of (3.80) is an eigenfunction with eigenvalue $2 \cos v q$ for the operator (3.76), viewed as a discrete difference operator on the Hilbert space (3.82). 
An interpretation of the operators (3.77) and (3.78) as Hilbert space operators is less straightforward. We see only one sensible way to do this without loosing formal self-adjointness: Both $\mathcal{X}$ and $\hat{\mathcal{C}}$ should be finite-dimensional. (This can also be understood from the fact that the natural classical $\mathrm{III}_{\text {rel, } b}$ phase space is a bounded subset of $\mathbf{R}^{2 \mathrm{~N}-2}$ [8].) More in detail, it appears inevitable to impose a quantization constraint on the parameters $\beta, v, g \in(0, \infty)$ : They should satisfy

$$
2 \tau+l \alpha=\pi, \quad \alpha \equiv \beta v, \quad \tau \equiv \alpha g, \quad l \in \mathbb{N}^{*}
$$

and then the Hilbert spaces are given by

$$
\Re=l^{2}(G), \quad \hat{\mathcal{K}}=l^{2}(\hat{G})
$$

where

$$
v G=\beta \hat{G}=\{\tau, \tau+\alpha, \ldots, \tau+l \alpha\} \equiv M(l, \tau)
$$

Indeed, restricting $q$ and $\theta$ to the points in $G$ and $\hat{G}$, resp., given by

$$
q_{m} \equiv \beta g+\beta m, \quad \theta_{n} \equiv v g+v n, \quad m, n=0, \ldots, l,
$$

the operators (3.77) and (3.78) have a well-defined self-adjoint action on $\mathcal{X}$ and $\hat{\mathcal{C}}$, resp.

It so happens that the eigenfunctions of the operators just defined are again already known, in essence. Indeed, now one has

$$
E\left(q_{m}, \theta_{n}\right)=\left[w\left(v q_{m}\right) w\left(\beta \theta_{n}\right)\right]^{\frac{1}{2}} P_{n}\left(\cos v q_{m}\right), \quad m, n=0, \ldots, l
$$

where $w$ is the weight function

$$
w(\tau+k \alpha) \equiv \frac{\sin (\tau+k \alpha)}{\sin \tau} \prod_{j=0}^{k-1} \frac{\sin (l-j) \alpha}{\sin (j+1) \alpha}, \quad k=0, \ldots, l
$$

on $M(l, \tau)$ and the $P_{n}$ are corresponding orthogonal polynomials. These may be viewed as special cases of discrete $q$-polynomials and corresponding weight functions obtained in [49]. Specifically, the relation of the Askey-Wilson parameters [49] to ours can be taken to be

$$
N \rightarrow l, \quad q \rightarrow \exp (i \alpha), \quad a=b=-c=-d \rightarrow \exp (i \tau-i \alpha / 2)
$$

and then one obtains

$$
p_{n}(\mu(m)) \rightarrow p_{n}(2 \exp (i \tau) \cos (\tau+m \alpha)) \equiv c P_{n}(\cos (\tau+m \alpha))
$$

where $c$ is a positive normalization constant chosen such that (3.89) defines an orthogonal matrix. The self-duality of the classical III $_{\text {rel, }, b}$ regime is again preserved under quantization, since one has [49]

$$
P_{n}(\cos (\tau+m \alpha))=P_{m}(\cos (\tau+n \alpha))
$$

For $g \in \mathbb{N}$ the desired eigenfunctions for the $N=2 \mathrm{III}_{\mathrm{rel}}$ and $\mathrm{III}_{\mathrm{rel}, b}$ cases can also be obtained via analytic continuation of our explicit $\mathrm{II}_{\text {rel }}$ solutions (3.38)-(3.42). The representations of the abovementioned orthogonal polynomials that arise in this way are new. In this connection it is to be noted that the various representations for the $q$-Gegenbauer polynomials in terms of basic hypergeometric functions (in which the index $n$ can be taken to be complex) do not admit a continuation to the $\mathrm{II}_{\text {rel }}$ regime. The difficulty at issue here has already been discussed in Subsection 3B2, in another guise: One would have to factor off (unknown) periodic functions that obstruct analytic continuation.

Next, let us briefly sketch what is known concerning arbitrary $N$ eigenfunctions. First, the work of Heckman and Opdam [34-37] mentioned before yields (on specialization to $A_{N-1}$ ) a complete solution for the $\mathrm{III}_{\mathrm{nr}}$ case. The joint eigenfunctions can be written (in the center-of-mass system) as products of factorized weight functions and polynomials in $N-1$ variables. The Plancherel problem is now much simpler than for the $\mathrm{II}_{\mathrm{nr}}$ case, since one is dealing with orthogonal polynomials. It has been solved (for arbitrary root systems) by Heckman [35]. 
The answers to (most of) the analogous questions for the III $_{\text {rel }}$ regime can be found in recent work by McDonald [50]. He also considers arbitrary root systems, and for $A_{N-1}$ his fundamental shift operator may be viewed as a transform of our operator $S_{1}$ (with $q_{1}+\cdots+q_{N}=0$ ). The structure of the joint eigenfunctions following from his work is the same as for the $\mathrm{II}_{\mathrm{nr}}$ case. (His work and the connection to our commuting III $_{\text {rel }}$ operators were pointed out to us by $\mathrm{T}$. Koornwinder [48].)

As concerns eigenfunctions for the III $_{\mathrm{rel}, b}$ case with $2<N<\pi / \tau$ (recall (2.94)), there are only speculations: We expect that they can be obtained via analytic continuation of McDonald's polynomials, and that they will turn out to be self-dual.

Finally, we would like to describe some results by T. Koornwinder that have a bearing on the $\mathrm{III}_{\text {rel }}$ eigenfunctions. First, he has proven [48] that they have the duality properties expected from the classical level, by means of an induction argument which applies to all root systems considered in [50]. Second, he has tied in the $q$-Legendre polynomials (i.e., the $q$-Gegenbauer polynomials with $g=1 / 2$ ) with Woronowicz' impressive work on compact quantum groups [51-53]. Specifically, he has shown [54] that they may be viewed as zonal spherical polynomials associated with Woronowicz' version of $S_{q} U(2)$ [51,52], as a natural $q$-analog of the relation between Legendre polynomials and $S U(2) \simeq S_{1} U(2)$. However, the definition of 'spherical' is no longer unambiguous for $q \in(0,1)[54]$. It can be expected that McDonald's $g=1 / 2 A_{N-1}$ polynomials may be similarly tied in with harmonic analysis on $S_{q} U(N)[52,53]$.

3C3. Elliptic systemss. As already mentioned, there is no guarantee that one can find a quantization of a classical integrable system for which commutativity is preserved. However, no ordering ambiguities occur when one quantizes the symmetric functions of $L\left(\mathrm{IV}_{\mathrm{nr}}\right)$, cf. (2.96), and the resulting operators do commute. This is proven by Olshanetsky and Perelomov [5] in the same way as sketched above for the $\mathrm{II}_{\mathrm{ar}}$ case.

Next, let us consider the $\mathrm{IV}_{\text {rel }}$ case. It so happens that there again exists a factorization of the potential yielding commuting operators $S_{1}, \ldots, S_{N}$. This factorization involves the Weierstrass $\sigma$ function. Specifically, if one replaces (3.6) by

$$
f_{ \pm}^{2}(q) \equiv \sigma(q \pm \gamma) / \sigma(q), \quad \gamma \equiv i \beta g
$$

then the operators (3.5) commute. This follows from the fact that the functional equations (3.7) still hold true when sh is replaced by $\sigma$ [2].

The latter functional equations are the most general expression of complete integrability for all of the systems considered in this survey (except possibly for the quantum $\mathrm{IV}_{\mathrm{nr}}$ systems, cf. below). Indeed, involutivity for the classical $\mathrm{IV}_{\text {rel }}$ case (and hence for all classical cases I-VI) finds its expression in a sequence of functional equations for the $\odot$-function that arise when one divides the $\sigma$-analog of (3.7) by $\rho$ and sends $\rho$ to 0 . (In fact, this is the only known proof of Liouville integrability for the $\mathrm{IV}_{\text {rel }}$ case, thus far.) Moreover, quantum commutativity for the relativistic cases follows by taking limits (the $\mathrm{V}_{\mathrm{rel}}$ case will be detailed in the next subsection). This entails commutativity for the cases $\mathrm{II}_{\mathrm{nr}}$ and $\mathrm{VI}_{\mathrm{nr}}$ by virtue of the reasoning in Subsection 3B1. Then the cases $\mathrm{I}_{\mathrm{nr}}$ and $\mathrm{III}_{\mathrm{nr}}$ follow from $\mathrm{II}_{\mathrm{nr}}$. The $V_{n r}$ case will be dealt with in the next subsection.

Unfortunately, we have no complete proof that commutativity for the quantum $\mathrm{IV}_{\mathrm{nr}}$ case may be obtained as a corollary. When one replaces $S_{l}(\beta)$ in (3.8) by $\Sigma_{l}(\beta)$ (given by $(2.101),(2.102)$ ), then the difficulty is that the coefficients of the $T_{k, i}$ (cf. the reasoning below (3.15)) might be non-zero constants, due to functional relations between the various functions involved. (Note that any such constant must vanish when $\omega$ or $\omega^{\prime}$ is taken to $\infty$.) However, for $k=1,2,3$ one does obtain $n_{k}=k$, cf. the explicit formulas (3.33)-(3.35) in [2], and we believe that, more generally, $n_{k}=k$ for $k \leqslant N$.

If one could prove this conjecture, then one would obtain (3.16), (3.17). Note, however, that the $k=2,3$ formulas just cited entail that $A_{2}^{(2)}$ and $A_{3}^{(3)}$ are not equal to the second and third symmetric function, resp., of the quantized Lax matrix (2.96) with $g$ replaced by $[g(g-1)]^{1 / 2}$.

At this point we would like to repeat that the fundamental role of the functional equations (3.7) for the $\sigma$-function pertains to the root system $A_{N-1}$ considered throughout this survey. However, the 
obvious conjecture is now, that it should be possible to generalize the functional equations to other root systems, thus yielding quantum and classical integrable systems generalizing those considered in $[4,5]$.

Finally, let us turn to explicit eigenfunctions. Since (3.19) still holds at the elliptic level (recall (3.94)), we shall take $g \neq 0,1$. Then the eigenfunctions are only known for $N=2$ and $g=2,3,4, \cdots$. Specifically, the relevant IV $_{\mathrm{nr}}$ operators read

$$
H_{\mathrm{nr}}=-\frac{d^{2}}{d q^{2}}+g(g-1) \mathscr{P}(q), \quad g=2,3, \cdots \quad\left(\mathrm{IV}_{\mathrm{nr}}\right)
$$

Their eigenfunctions are the well-known Lamé functions [28]. The relativistic $A \Delta O$ s generalizing $H_{\mathrm{nr}}$ read

$H=\left(\frac{\sigma(q-i \beta g)}{\sigma(q)}\right)^{-\frac{1}{2}} T_{i \beta}\left(\frac{\sigma(q+i \beta g)}{\sigma(q)}\right)^{\frac{1}{2}}+(\beta \rightarrow-\beta), \quad g=2,3, \cdots \quad\left(\mathrm{IV}_{\mathrm{rel}}\right)$

For these $A \Delta O$ s we have found explicit eigenfunctions reducing to the Lame functions in the nonrelativistic limit $\beta \rightarrow 0$. Details concerning these functions and their Hilbert space properties will appear elsewhere [55].

3C4. Periodic Toda type systems. When one quantizes the Hamiltonian (2.106) and the symmetric functions of the Lax matrix (2.107) associated with the periodic Toda systems $V_{n r}$, then the resulting operators commute. This can be proven in the same way as for the case $\mathrm{II}_{\mathrm{nr}}[5]$. For the relativistic case $V_{\text {rel }}$ one encounters the same ordering ambiguities as for the case $\mathrm{VI}_{\mathrm{rel}}$. However, the same ordering choice as made in (3.18) (but now with (2.109), (2.110) in force) yields commuting operators $H, S_{1}, \ldots, S_{N}$. This can be proven directly [3], but it is also possible to obtain this as a corollary of the quantum $I V_{\text {rel }}$ commutativity. Indeed, we may replace the $\sigma$-functions in the quantum $I V_{\text {rel }}$ operator $S_{l}$ (given by (3.5), (3.94)) by the rhs of (2.111) with the first exponential omitted, since these exponentials only give rise to an overall multiplicative constant in $S_{l}$. If we then substitute (2.112) and (2.118), and take $\omega \rightarrow \infty$, we obtain the $\mathrm{V}_{\text {rel }}$ operator (3.18); The ' $\mathrm{VI}_{\text {rel }}$ part' comes from the sh-factor at the rhs of (2.111), whereas the infinite product supplies the extra terms needed to turn the $\mathrm{VI}_{\text {rel }}$ into the $V_{\text {rel }}$ operator. In a similar fashion, the $\sigma$-analogs of the functional equations (3.7) turn into the functional equations expressing $V_{\text {rel }}$ quantum commutativity [3].

When one substitutes $(2.108)$ in the $V_{\text {rel }}$ operators and takes $\tau$ to 0 , then one obtains the $\mathrm{VI}_{\text {rel }}$ operators, as is easily verified.

The $\mathrm{V}_{\mathrm{nr}}$ transition involves more work. When we start again from (3.8), with $S_{l}(\beta)$ replaced by the $V_{\text {rel }}$ operators derived from $\Sigma_{l}$, cf. (2.126), then the reasoning below (3.15) can only be followed til the point where interparticle distances are taken to $\infty$. There is no analog of this for the $V_{\text {rel }}$ case, since the functions $e_{j} \equiv \exp \left[\mu\left(q_{j}-q_{j-1}\right], j=1, \ldots, N\right.$, cannot simultaneously go to 0 . Indeed, this is clear from the relation $e_{1} \cdots e_{N}=1$. Even so, we can again obtain a contradiction, as follows.

For $n_{k}<k$ all terms in $A_{k}^{\left(n_{k}\right)}$ are of the form $P\left(e_{1}, \ldots, e_{N}\right) \partial_{1}^{\alpha_{1}} \cdots \partial_{N}^{\alpha_{N}}$, where $0 \leqslant|\alpha| \leqslant n_{k}-1$ and $P$ is a polynomial that has no constant term. But the coefficients of the terms $T_{k, i}$ must be constant by virtue of the arguments below (3.15). Since these coefficients are of the form $P\left(e_{1}, \ldots, e_{N-1}, 1 / e_{1} \cdots e_{N-1}\right)$, where $P$ is a polynomial without constant term, we must have $P\left(x_{1}, \ldots, x_{N}\right)=\sum_{j=1}^{M} c_{j}\left(x_{1} \cdots x_{N}\right)^{j}$. But the minimal order in $\beta$ at which such contributions to $A_{k}(\beta)$ can arise equals $2 N$. Since $n_{k}<k \leqslant N$ by assumption, we arrive at a contradiction.

The upshot is, that we must have $n_{k}=k$, and hence the desired relations (3.16), (3.17) follow. Moreover, we may conclude that $A_{k}^{(k)}$ equals the $k$-th symmetric function of the quantized Lax matrix (2.107). Indeed, the difference commutes with $H_{\mathrm{nr}}$ [5] and consists of terms that arise when partials act on potentials. Therefore, the above arguments can be used once more.

Finally, let us describe the state of the art as regards joint eigenfunctions. This is quite similar to the nonperiodic case: For $V_{\text {rel }}$ one is dealing with non-self-adjoint operators and nothing is known, 
whereas for $V_{n r}$ the eigenfunctions have been found and studied in considerable detail (by Goodman and Wallach [56]).

\section{CONNECTIONS WITH INFINITE-DIMENSIONAL INTEGRABLE SYSTEMS}

\section{A. Preamble}

In recent years it has been widely advertised that there are intimate relations between infinitedimensional integrable system theory and various subdisciplines of mathematics and physics that would appear to be rather far removed from this area at first sight. The latter include the representation theory of Kac-Moody and Virasoro algebras and generalizations thereof, soluble models in twodimensional classical and one-dimensional quantum statistical mechanics, quantum group theory, knot theory, conformally invariant field theories, string theories, . . . A recent 'flow chart' of the interconnections between the various fields can be found in [57]. This flow chart is quite extensive and the fields it covers are currently investigated by large numbers of researchers.

Our purpose in this chapter may be phrased as adding yet another box to the flow chart of [57], for which we propose the label 'Finite-dimensional Soliton Systems'. Some of the contents of this box have been sketched in the previous two chapters, but it should be repeated that we have restricted ourselves to the root system $A_{N-1}$, absence of integrable external field couplings and internal degrees of freedom, and zero temperature. We shall continue to do so in this chapter.

\section{B. THE CLASSICAL LEVEL}

The $\mathrm{II}_{\text {rel }}$ systems are intimately connected to the pure soliton solutions of various soliton PDEs and infinite soliton lattices. The latter include the sine-Gordon equation, the $A_{n}^{(1)}$-reductions of the $K P$ equation (yielding $\mathrm{KdV}$, Boussinesq, ... for $n=1,2, \cdots$ ), the modified $\mathrm{KdV}$ equation, the infinite Toda lattice, .... Similarly, solutions describing solitons, antisolitons and their bound states can be obtained from the $\tilde{\mathrm{I}}_{\text {rel }}$ systems. The latter situation is discussed in [7]; here, we shall restrict ourselves to the pure soliton case $[1,58]$.

We begin by recalling that we have already considered quite general one-parameter flows associated with the $\mathrm{I}_{\text {rel }}$ systems, cf. (2.63)-(2.69). Here, we have occasion to study two-parameter flows. Thus, we now work with

$$
\hat{A}\left(1,1, z ; \hat{q}_{1}+t h_{0}{ }^{\prime}\left(\hat{\theta}_{1}\right)-x h_{1}{ }^{\prime}\left(\hat{\theta}_{1}\right), \ldots, \hat{q}_{N}+t h_{0}{ }^{\prime}\left(\hat{\theta}_{N}\right)-x h_{1}{ }^{\prime}\left(\hat{\theta}_{N}\right), \hat{\theta}\right)
$$

(where $h_{0}, h_{1}$ are real-valued) instead of (2.69). If we then set

$$
H_{j} \equiv \operatorname{Tr} h_{j}(\ln L) \quad j=0,1
$$

and

$$
q(t, x) \equiv \exp \left(t H_{0}-x H_{1}\right)(q, \theta)_{\text {conf. }}
$$

(where conf. denotes the projection on configuration space), it follows as before that the eigenvalues of $(4.1)$ are given by $\exp \left[q_{1}(t, x)\right], \ldots, \exp \left[q_{N}(t, x)\right]$. Therefore, one may conclude

$$
\begin{aligned}
& \ln (\operatorname{det}(1+\hat{A}))=\sum_{j=1}^{N} \ln \left(1+\exp \left[q_{j}(t, x)\right]\right) \\
& \operatorname{Tr}(\operatorname{Arctg} \hat{A})=\sum_{j=1}^{N} \operatorname{Arctg}\left(\exp \left[q_{j}(t, x)\right]\right) .
\end{aligned}
$$

The crux is now, that the functions at the rhs or certain derivatives thereof are pure soliton solutions to the above-mentioned soliton PDEs for an appropriate choice of $h_{0}$ and $h_{1}$ and of the "coupling constant' $z$. (For infinite lattices one should take $x \in \mathbb{Z}$.) The details are spelled out in [1,58].

As a consequence of the formulas (4.4), (4.5) one may view soliton solutions to the abovementioned infinite-dimensional integrable systems as linear superpositions of single soliton terms. 
Each of these terms gives rise to a uniquely determined space-time trajectory $x_{j}(t)$, obtained via the requirement $q_{j}\left(t, x_{j}(t)\right)=0$. The asymptotics of these trajectories is the same as for the soliton.solutions $[58,7]$. In this way an intuitive picture suggests itself of solitons being deformations of an elastic medium that conceals an underlying point particle dynamics.

We proceed by discussing an issue that is relevant to the problem of quantizing those infinitedimensional dynamical systems whose particle-like solutions can be obtained from the $N$-particle systems discussed in Chapter 2. This concerns Hamiltonian formulations of the Inverse Scattering Transform (IST) for the former systems. Such formulations lead to soliton action-angle coordinates that can be compared to the variables $\hat{q}, \hat{\theta}$, cf. [1, Subsection $6 \mathrm{D}]$. The result is, that the angle variables coincide (in essence) with $\hat{q}_{1}, \ldots, \hat{q}_{N}$ for the (m)KdV, sine-Gordon and Toda cases, whereas $\hat{\theta}_{1}, \ldots, \hat{\theta}_{N}$ coincide with the soliton action variables only for the $\mathrm{mKdV}$ and $s G$ cases. For the $\mathrm{KdV}$ and Toda cases the action variables of $[59,60]$ and $[61,62]$, resp., are given by

$$
\begin{aligned}
& p_{j}=\exp \left(2 \hat{\theta}_{j}\right) \\
& p_{j}=-2 \operatorname{cth}\left(\hat{\theta}_{j}\right)
\end{aligned}
$$

To specify and discuss the soliton $S$-map for these two cases, it is expedient to first identify $\Omega^{-}$ with $\Omega^{+} \simeq \Omega$ in the $\mathrm{II}_{\mathrm{rel}}$ equations (2.74)-(2.77) (via reversal of ordering). Moreover, we put $\beta=\mu=1$ in the latter and denote the $S$-map on $\Omega^{-} \simeq \hat{\Omega}$ thus obtained by $S(z)$. Then the soliton $S$-map for the $\mathrm{KdV}$ and Toda cases (with the parametrizations (4.6) and (4.7), resp., in force) is given by $S$ ( $i \pi / 2$ ), cf. [1]. Now one readily verifies that $S(z)$ is canonical w.r.t. a symplectic form

$$
\hat{\omega} \equiv \sum_{j=1}^{N} d \hat{q}_{j} \wedge d p\left(\hat{\theta}_{j}\right)
$$

if and only if $p(\theta)$ is linear in $\theta$. In particular, the $\mathrm{KdV}$ and Toda $N$-soliton $S$-map $S(i \pi / 2)$ is not canonical when one employs $\hat{q}_{j}$ and $p_{j}$ (given by (4.6) and (4.7), resp.) as canonical coordinates on the scattering data. (In the Toda case the $j$-th angle variable used in $[61,62]$ is not equal to $\hat{q}_{j}$, but the difference depends only on $\hat{\theta}_{j}$, so our arguments do apply.)

The violation of canonicity just established may not look startling at first sight. However, it does appear bizarre when viewed from another perspective. Indeed, one would be inclined to expect that the asymptotics of a Hamiltonian (and hence canonical) flow is coded in an $S$-map that is also canonical. (On a personal note we might add that we were very puzzled when we noticed the non-canonicity of the KdV soliton $S$-map some ten years ago. When we asked $H$. Segur for advice, he was not puzzled: He simply did not believe us!)

We would like to clarify this issue here, since it has a bearing on the soliton $\simeq$ particle correspondences sketched above. (We should mention at this point that the non-canonicity of soliton $S$-maps for the KdV, Toda and finite-density nonlinear Schrödinger (NLS) cases has been observed and discussed in [62,63]. However, we feel that both the diagnosis and the remedy presented in [63] are far from compelling.)

First and foremost, it should be recalled that neither the IST nor the soliton $S$-map involve any Poisson or symplectic structures. Such a structure is needed only when one wishes to write the nonlinear evolution in Hamiltonian form. More specifically, the equation of motion gives rise to a vector field $X$ on the manifold $\Omega$ of Cauchy initial values, and the structure then ensures that $X$ may be viewed as the vector field associated with a Hamiltonian $H$ on $\Omega$. Now the direct transformation $\Phi$ from $\Omega$ onto the manifold $\hat{\Omega}$ of scattering data transforms $X$ into a vector field $\hat{X}$ on $\hat{\Omega}$, whose flow is linear on one half of the variables coordinatizing $\hat{\Omega}$ and trivial on the other half. In such circumstances there are uncountably many different symplectic forms $\hat{\omega}$ on $\hat{\Omega}$ such that $\hat{X}$ is the vector field arising via $\hat{\omega}$ from a Hamiltonian $H$ on $\hat{\mathbf{\Omega}}$. A finite-dimensional example (which encodes the $\mathrm{KdV}$ $N$-soliton situation) may be in order: With $\hat{\Omega}$ equal to (2.51) one may take

$$
\hat{X}=\sum_{j=1}^{N} \exp \left(3 \hat{\theta}_{j}\right) \partial_{\hat{q}_{j}}, \quad \hat{\omega}=\sum_{j=1}^{N} f\left(\hat{\theta}_{j}\right) d \hat{q}_{j} \wedge d \hat{\theta}_{j}, \quad \hat{H}=\sum_{j=1}^{N} \int_{-\infty}^{\hat{\theta}_{j}} d u f(u) \exp (3 u)
$$


for any nonvanishing $f$ such that the integral makes sense.

Of course, one wants $\hat{\omega}$ and $\hat{H}$ to be smooth in a suitable sense, but in the absence of a clear picture of the topology of the scattering data manifold $\hat{\Omega}$ it is impossible to pin down just what restrictions this entails. In fact, to proceed we shall also assume that $\Phi$ is 'smooth'. (Again, this has not been sorted out with the standards of rigor that are taken for granted in modern global analysis, as far as we know.) Accepting this, it is obvious that the pullbacks under $\Phi$ of the above-mentioned forms $\hat{\omega}$ and Hamiltonians $H$ give rise to symplectic forms $\omega$ on $\Omega$ (since ' $d$ commutes with pullback') and Hamiltonians $H$ that all lead to the same vector field $X$ on $\Omega$ one started with.

The upshot is, that there exist uncountably many different Hamiltonian formulations of the IST. Let us now explain why the soliton $S$-map need not be canonical for any of these formulations, a priori. This becomes evident when soliton scattering theory is formulated in a mathematically precise way. Such a formulation should involve a comparison map $J: \hat{\Omega} \rightarrow \Omega$ that identifies a point in $\hat{\Omega}$ with $N$ bound states and vanishing reflection with a function in $\Omega$ that equals a linear superposition of $N$ one-soliton functions depending on the $N$ energies and norming constants. (Cf. [12, Subsection 2F] for more details on this picture.) Since one is comparing two very different symplectic manifolds (intertwined by the IST), it is in fact extremely optimistic to expect that $J$ will be asymptotically canonical, in the sense that the wave transformations in the two-space scattering theory picture [10,12] not only exist, but are also canonical. However, if the wave maps are not canonical, then $S$ is not likely to be canonical.

On the other hand, whenever one succeeds in parametrizing soliton solutions with angle parameters $\hat{q}_{1}, \ldots, \hat{q}_{N}$ and action parameters $\hat{\theta}_{1}, \ldots, \hat{\theta}_{N}$ in such a way that the soliton $S$-map is given by $S(z)$, $-i z \in(0, \pi / 2]$, then the $S$-map is canonical w.r.t. the symplectic form (4.8), provided one takes $p(\theta)$ linear in $\theta$. Then the soliton part of the Hamiltonian is (in essence) uniquely determined (cf. (4.9) for the $\mathrm{KdV}$ case). Of course, the radiation should be taken into account as well, but it so happens that this can be done in a very natural way for the cases at hand.

Indeed, let us show this first for the $\mathrm{KdV}$ case, where we need

$$
\hat{H}=\frac{1}{3} \sum_{j=1}^{N} p_{j}^{3 / 2}=\frac{1}{3} \sum_{j=1}^{N} \exp \left(3 \hat{\theta}_{j}\right)
$$

when we use the form (2.52), cf. (4.9). This corresponds to the soliton part of the Hamiltonian

$$
H=\frac{1}{2} \int d x u^{2}(x)
$$

on $\Omega$, i.e., the Hamiltonian $-I_{1}$ in the well-known hierarchy. Therefore, one gets a smooth extension of $\hat{H}$ to all of $\hat{\Omega}$ by picking it equal to $-I_{1} \circ \delta$, where $\varepsilon=\Phi^{-1}$ denotes the IST. The requirement that the Hamiltonian $H$ thus obtained generate the $\mathrm{KdV}$ evolution of the reflection coefficient (as found via the direct transformation) now fixes the symplectic form on the radiation, in the following sense. When we keep the customary angle variables $\varphi(k)(\sim$ the arguments of the reflection coefficient), then the new action variables $\tilde{\rho}(k)$ are uniquely determined by the evolution requirement just made and by insisting that

$$
\{\varphi(l), \tilde{\rho}(k)\}=\delta(k-l), \quad\left\{\psi(k), \hat{x}_{j}\right\}=0, \quad \psi=\phi, \tilde{\rho}, \quad x=q, \theta .
$$

Proceeding in this way, we find

$$
\tilde{\rho}(k)=\left(4 k^{2}\right)^{-1} \rho(k)
$$

when $\rho(k)$ and $\varphi(k)$ are given by [63, Eq. (8)]. With the symplectic form thus fixed, one readily verifies that the complete $S$-map (solitons + radiation), as specified in [63, Eqs. (21), (22)], is canonical. (Note that the $q_{k}$ employed in 1.c. corresponds to $-\hat{q}_{k} / 2$.) Furthermore, the higher flows in the hierarchy are now generated by $-I_{2 n-1}$ instead of $I_{2 n+1}$ (for $n>0$ ), whereas the Zakharov-Faddeev momentum Hamiltonian $I_{1}$ should be replaced by 


$$
P=-\frac{1}{2} \int d x u(x)
$$

(Here, the sign conforms to the sign in the KdV equation $\dot{u}-6 u u_{x}+u_{x x x}=0$ employed in most of the literature.)

The latter facts make it very plausible that the new symplectic structure on $\Omega$ just defined coincides with the 'second Hamiltonian structure' introduced by Magri [64,65] (up to a sign that corresponds to the different sign of our angle variables $\hat{q}_{j}$ compared to the angle variables used in the literature). The reason that we are not sure that (minus) Magri's structure results is that we are requiring that soliton and radiation variables commute, cf. (4.12). To our knowledge, the relations (4.12) have not been proven for Magri's structure; possibly, terms propotional to $\delta(k)$ are present. Such terms must occur for the 'first Hamiltonian structure' (Gardner's structure [66]), since otherwise blatant contradictions arise [67]. (We feel that the presence of such terms is a highly undesirable feature of the Gardner choice.)

We can see only two drawbacks of the new symplectic structure: It is far less 'obvious' than Gardner's structure (even when it does equal Magri's structure up to sign), and it is not likely to admit an $r$-matrix formulation, in contrast to Gardner's structure (cf. [62, p.467]). However, in our opinion these liabilities of the new structure are negligible compared to its assets: (i) By definition, it decouples soliton and radiation modes; (ii) It gives rise to a canonical scattering map; (iii) It obviates certain unphysical characteristics of the energy and momentum Hamiltonians associated with the Gardner choice.

To substantiate the latter claim, we point out that the Gardner energy (momentum) has opposite (the same) sign for radiation and soliton solutions. In contrast, the energy (4.11) is always positive (as expected for any disturbance), and the momentum (4.14) is negative/positive for radiation/solitons (as should be the case, since radiation/soliton solutions move from right to left/left to right [68]).

Next, let us consider the Toda case. When one combines [62, p. 504, Eq. (4.42)] with the parametrization $z_{j}=\operatorname{th}\left(\hat{\theta}_{j} / 2\right)$ corresponding to [1, Eq. (6.17)], then one obtains $\hat{q}_{j}=2 / \mathrm{sh} \hat{\theta}_{j}$, cf. also [1, Eqs. (6.6), (6.18)]. Thus, the Hamiltonian corresponding to the choice (2.52) reads

$$
\hat{H}=\sum_{j=1}^{N} \ln \left(z_{j}^{2}\right)=-\sum_{j=1}^{N} \ln \left(\mathrm{cth}^{2} \frac{1}{2} \hat{\theta}_{j}\right) \quad \text { (Toda). }
$$

Again, there is an obvious way to extend to the radiation: If one compares with [62, p. 504, Eq. (4.36)], one sees that $\hat{H}$ may be viewed as the transform of the 'total elongation' functional

$$
H=-\lim _{n \rightarrow \infty} q_{n}
$$

Therefore, we can proceed just as for the $\mathrm{KdV}$ case: We require (4.12) and then take

$$
\tilde{\rho}(\theta)=\left(2 \sin ^{2} \theta\right)^{-1} \rho(\theta)
$$

where $\rho(\theta)$ is given by [62, p. 503, Eq. (4.28)]. This ensures that $\phi(\theta)$ satisfies $\dot{\phi}=2 \sin \theta$, as desired (cf. $[62$, p. 504, Eq. (4.41)]). There appears to be no obvious candidate for the new symplectic structure on $\Omega$ that corresponds to the symplectic structure on $\hat{\Omega}$ just defined.

Admittedly, the new structure and the corresponding Hamiltonian (4.16) are not exactly the obvious ones for the Toda lattice. Possibly, the situation can be better understood by answering the analogous questions for the relativistic Toda lattice, and then taking the speed of light to infinity. Again, there are an 'obvious' Hamiltonian and symplectic structure that arise from viewing the lattice as the $N \rightarrow \infty$ limit of the $\mathrm{V}_{\text {rel }}$ (or $\mathrm{VI}_{\text {rel }}$ ) systems, cf. Chapter 2. Moreover, an IST formulation and $N$-soliton solutions are known [29]. However, the soliton $S$-map has not been determined yet.

As we shall argue in the next section, the quantized nonlinear Schrodinger equation (NLS) may be viewed (in more than one way) as a degenerate case of the quantized particle systems of Chapter 3. We suspect that the classical NLS breathers (i.e., the solitons in the attractive and rapidly decreasing case [62]) may be tied in with degenerate particle systems, too. However, their explicit space-time dependence (as specified e.g. in [62, p. 132, Eq. (5.38)]) appears incompatible with (4.4) or (4.5). This 
also holds true for the repulsive finite-density NLS soliton solutions detailed in [62, p. 170, Eq. (8.33)].

On the other hand, we have found a clearcut connection of the latter solitons to the $\mathrm{II}_{\text {rel }}$ systems at the level of the $S$-map. Just as for the $\mathrm{KdV}$ and Toda cases, the soliton $S$-map is not canonical w.r.t. the symplectic structure arising via the $r$-matrix formulation (cf. [62, p. 266]). However, this can again be remedied by changing the symplectic form on $\hat{\Omega}$. Indeed, as the analog of the reparametrizations (4.6), (4.7) one now needs

$$
p_{j} \equiv \frac{\omega}{k} \text { th } \hat{\theta}_{j} \quad \text { (f.d. NLS). }
$$

Then the soliton $S$-map is again given by $S(i \pi / 2)$ (as is readily verified from [62, p. 266]), and hence is canonical w.r.t. the symplectic form (2.52). In the same way as for the $\mathrm{KdV}$ and Toda cases we now infer that we should take

$$
\hat{H}=\sum_{j=1}^{N} \omega\left(\omega^{2}-x^{2} p_{j}^{2}\right)^{1 / 2}=\sum_{j=1}^{N} \omega^{2} / \operatorname{ch} \hat{\theta}_{j} \quad \text { (f.d. NLS) }
$$

as new soliton Hamiltonian, cf. [62, p. 264, Eqs. (9.100), (9.101)]. Comparing to [62, p. 258, Eq. (9.64)], it follows that $\hat{H}$ can be smoothly extended to radiation by taking

$$
H=-\kappa \omega J_{1, \rho} \quad \text { (f.d NLS) }
$$

as the analog of (4.11) and (4.16). When we now insist on (4.12), then we need

$$
\tilde{\rho}(\lambda)=\kappa \omega\left(\omega^{2}-\lambda^{2}\right)^{-1} \rho(\lambda)
$$

with $\rho(\lambda)$ and $\varphi(\lambda)$ given by $[62$, p. 254 , Eq. (9.8)], in order to obtain the NLS evolution $\dot{\varphi}=\lambda\left(\lambda^{2}-\omega^{2}\right)^{1 / 2}$, cf. [62, p. 145, Eq. (6.51)].

The state of affairs for the finite-density NLS case we have just sketched is surprisingly similar to that for the Toda and KdV cases. The orthodox Hamiltonians and symplectic structures give rise to non-canonical soliton scattering and an unwanted coupling between radiation and solitons, cf. [62, $\mathrm{p}$. 257, p. 503] and [63], resp. However, there exist a Hamiltonian $\hat{H}$ 'lower down' in the usual hierarchy and a symplectic form $\hat{\omega}$ on the scattering data manifold $\hat{\Omega}$ that are essentially unique when three requirements are imposed: (i) The soliton and radiation variables should commute; (ii) $\hat{H}$ and $\hat{\omega}$ should give rise to the vector field $\hat{X}$ on $\hat{\mathbf{\Omega}}$ that follows from the direct transformation; (iii) The soliton $S$-map should be canonical.

The acid test for the unorthodox structures obeying these requirements now reads: Is the complete $S$-map also canonical? We have already seen above that the answer is 'yes' for the KdV case. We conjecture that the answer is affirmative for the other two cases, too. (It seems the complete $S$-map is not known for these two models.) Even when this can be proven, the new structures and Hamiltonians (4.16), (4.20) remain puzzling. Possibly, the need for a change of the 'obvious' structure and Hamiltonian arises from a rigorously controlled infinite volume limit. In this connection it should be recalled that for nontrivial relativistic quantum field theories such limits always give rise to a unitarily inequivalent representation of the canonical (anti)commutation relations (Haag's theorem) and to a drastic change in the dynamics. This analogy may appear far-fetched at first sight, but it should be remembered that classical Hamiltonian systems can be formulated as quantum Hamiltonian systems of a very special kind by using the Koopman formalism; as is well known, canonicity amounts to unitarity in this formalism.

Of course, the correspondence between the pure soliton $N$-particle systems and the various soliton PDEs and lattices mentioned above has no direct bearing on the IST for the latter systems. Moreover, it cannot be extended to the radiation and 'mixed' solutions. (In this connection we would like to take issue with the assertion that particle-like initial values are dense. The topology in which this is supposed to be true is never specified, but if one exists, it has to be weaker than the $L^{1}(\mathbf{R})$-topology. Indeed, if $V_{n} \rightarrow V$ in $L^{1}(\mathbf{R}) \otimes C^{k}$ with $V_{n}$ reflectionless, then $V$ is reflectionless, too.)

On the other hand, as far as soliton $S$-maps are concerned, the particle systems appear to be more 
general. When appropriately parametrized, pure soliton solutions to completely different-looking evolution equations (including $\mathrm{sG}, \mathrm{mKdV}, \mathrm{KdV}$, Toda, finite-density NLS, . . ) all yield the $S$-map $S(i \pi / 2)$ of the hyperbolic $\mathrm{II}_{\mathrm{rd}}$ systems, whereas the $A_{n}^{(1)}$-reductions of the KP hierarchy lead to the $S$-maps $S(i \pi /(n+1))$ [58]. Moreover, the full panoply of particle-like solutions to the sG and mKdV equations can be modeled when one includes the $\tilde{\Pi}_{\mathrm{rel}}(z=i \pi / 2)$ particle systems [7].

In point of fact, there is also strong evidence that the particle-like solutions to the fully anisotropic Landau-Lifshitz equation lead to an $S$-map that occurs in our systems. The systems just referred to are the systems dual to the elliptic systems of Chapter 2, with $\gamma$ equal to $\omega^{\prime}$. Note this value is the elliptic analog of the value $z=i \pi / 2$ ): In the absence of an explicit action-angle map for the $I V_{\text {rel }}$ case, we do not know what these systems look like. This is a matter of considerable interest, and even more so when the quantion situation is taken into account.

The evidence we have in mind is the fact that it is possible to parametrize the particle-like LandauLifshitz solutions (as detailed in [69]) in such a way that the function $c_{12}$ of [69] can be written

$$
c_{12}=\frac{1}{4} k^{2}\left[\mathscr{T}\left(2 i K^{\prime} ; K, 2 i K^{\prime}\right)-\mathscr{P}\left(\hat{\theta}_{1}-\hat{\theta}_{2} ; K, 2 i K^{\prime}\right)\right]^{-1} \text {. }
$$

Specifically, defining the elliptic modulus by

$$
k \equiv\left(1-b^{2} / a^{2}\right)^{1 / 2}, \quad a>b>0
$$

and substituting

$$
k_{j}=-a \operatorname{dn}\left(\hat{\theta}_{j}, k\right) \quad(\mathrm{XYZ} \rightarrow \mathrm{XXZ})
$$

or

$$
k_{j}=-b \operatorname{nd}\left(\hat{\theta}_{j}, k\right) \quad(\mathrm{XYZ} \rightarrow \mathrm{sG})
$$

in $c_{12}$, one can verify that (4.22) holds true. Of course, these two substitutions must then be linearly related, and indeed one can get from (4.24) to (4.25) by shifting $\hat{\theta}_{j}$ over $K$. However, both are useful: One has

$$
\lim _{b \rightarrow 0} c_{12}=\operatorname{th}^{2} \frac{1}{2}\left(\hat{\theta}_{1}-\hat{\theta}_{2}\right)
$$

but the limit $b \rightarrow 0$ can only be taken in the complete solution [69, Eq. (3.12)] when one employs (4.24). This yields one of the two partially anisotropic cases, viz., the case where the two equal couplings are greater than the third one. To handle the $b \rightarrow 0$ limit when (4.25) is used, one must rescale $x$ and $t$ by a factor $b$, and then one can reach the particle-like sG solutions by proceeding as indicated in [62, pp. 459-460, Eqs. (8.15)-(8.19)].

It remains to explain the connection of (4.22) to soliton and particle $S$-maps. On the soliton side, the $S$-map seems not to be available in the literature. (We have not found any specification of soliton action-angle variables, either.) However, from [69, Eq. (3.12)] it is very plausible that the shifts of the soliton position parameters

$$
\hat{q}_{j} \equiv \operatorname{Re} \xi_{j}^{0}+\frac{1}{2} \ln \left(\prod_{k \neq j} c_{j k}\right)
$$

are factorized, with two-soliton shift given by $\ln \left(1 / c_{12}\right)$ (up to functions of the form $f_{j}\left(\hat{\theta}_{j}\right)$ ). On the particle side, asymptotics as just described result from the eigenvalue asymptotics of

$$
L\left(\operatorname{IV}_{\mathrm{red}}\right)\left(\hat{\theta}_{,} \hat{q}_{1}+t a_{1}, \ldots, \hat{q}_{N}+t a_{N}\right), \quad a_{N}<\cdots<a_{1}, \quad|t| \rightarrow \infty
$$

in the same way as sketched in Subsection 2B4. The point is, that if one takes the spectral parameter $\lambda$ equal to $\omega+\omega^{\prime}$, then one can show that a diagonal similarity transformation turns $L\left(I V_{\text {rel }}\right)$ into a positive matrix. Therefore, the asymptotics follows from [6, Th. A2] by using the generalized Cauchy identities of [2], yielding factorized shifts as just described. 


\section{C. THE QUANTUM LEVEL}

The particle picture of solitons that emerges from the considerations in the previous section is surprising and intriguing at the level of classical field theory, but not more than that from the viewpoint of physical applications. For instance, a solitary water wave is vastly different from a point particle. However, at the level of quantum field theory the physical status of a soliton-particle correspondence is quite different.

To see this, recall that the physical content of a (temperature and density zero) quantum field theory is completely determined by the $S$-operator and the bound-state spectrum. Quantum fields are auxiliary entities serving as a convenient vehicle to arrive at a theoretical understanding of experimental data concerning particle characteristics. We are not aware of alternative descriptions for quantum field theories in which particle annihilation and creation occurs. However, soliton quantum field theories such as the quantized sine-Gordon theory are characterized by an $S$-operator that conserves particle number. Therefore, any quantum dynamics that leads to the same $S$-operator (and boundstate spectrum) is physically indistinguishable from the quantum field dynamics.

We have worked out these considerations in more detail some ten years ago (cf. the Introduction of [13]). They served as motivation for the second part of [12], where we constructed relativistic particle dynamics leading to the $S$-operators of the Federbush and continuum Ising field theories. (To date, these are the only positive-energy relativistic quantum field theories for which not only nonperturbative existence, but also the soliton $S$-operator ascribed to them have been rigorously confirmed [70-74].) The $N$-particle dynamics involved in this alternative description (cf. [12, Section 3]) give rise to the simplest examples of what we have dubbed 'quantum pure soliton systems' in Section 3A, the $N$-particle $S$-matrix being given by the multiplication operator

$$
(S f)(\theta)=\prod_{1<j<k<N} \exp \left[i \phi \in\left(\theta_{j}-\theta_{k}\right)\right] f(\theta), \quad \phi \in[0,2 \pi)
$$

on $L^{2}\left(\mathbb{R}^{N}, d^{N} \theta\right)$. (Here and from now on, $\epsilon$ denotes the sign function.) As $N$-particle dynamics on $L^{2}\left(\mathbb{R}^{N}, d^{N} q\right)$ yielding this soliton $S$-operator one can choose

$$
H \equiv M(\cdot)\left(\sum_{j=1}^{N} \exp \left(\beta \hat{\theta}_{j}\right)\right) M(\cdot)^{*},
$$

where $\exp \left(\beta \hat{\theta}_{j}\right)$ denotes the Fourier transform of multiplication by $\exp \left(\beta \theta_{j}\right)$ (recall (3.1) and our standing convention $\hbar=1$ ), and where

$$
M(q) \equiv \prod_{1<j<k<N} \exp \left[i \phi \epsilon\left(q_{j}-q_{k}\right) / 2\right]
$$

(This is possibly not clear from [12, 1.c.], but can be gleaned from [75, Section 7B].)

It is to be noted that the classical version of $H$ is free, and that one may rewrite $H$ as

$$
H=\sum_{j=1}^{N} \prod_{k \neq j} \exp \left[i \phi \epsilon\left(q_{j}-q_{k}\right) / 2\right] \exp \left(\beta \hat{\theta}_{j}\right) \prod_{k \neq j} \exp \left[-i \phi \epsilon\left(q_{j}-q_{k}\right) / 2\right]
$$

Therefore, one may regard $H$ as a degenerate case of the operator $S_{1}\left(\mathrm{II}_{\mathrm{rel}}\right)$, obtained from (3.5), (3.6) by fixing $z$ and taking $\mu$ to $\infty$.

The limit just indicated amounts to taking $g$ to 0 with $\mu g$ fixed. Thus, even for $N=2$ we do not know whether this limit can be given a rigorous sense (in terms of strong convergence of eigenfunction transforms, say). At any rate, one would have to deal with eigenfunctions that are not invariant under parity (since (4.32) is not). When one takes the known $N=2 \mathrm{II}_{\mathrm{nr}}$ case as a lead, this is certainly not as preposterous as it may appear from (3.26) (which is formally invariant under parity). The point is, that the $\mathrm{II}_{\mathrm{nr}}$ Hamiltonian (3.34) is not essentially self-adjoint on $C_{0}^{\infty}\left(\mathbb{R}^{*}\right)$ for $g \in\left(\frac{1}{2}, \frac{3}{2}\right)$, and 'almost all' self-adjoint extensions will violate the formal parity invariance of (3.34).

The issue of self-adjoint extensions just mentioned is the key to the connection between the $\mathrm{II}_{\mathrm{nr}}$ systems and the quantized nonlinear Schrödinger (NLS) theory. We proceed to describe this relation. To 
this end, we first recall that the latter quantum field theory is characterized by an explicitly particle number preserving Hamiltonian. Specifically, the restriction to the $N$-particle sector reads

$$
H \equiv-\frac{1}{2} \Delta+\lambda \sum_{1<j<k<N} \delta\left(q_{j}-q_{k}\right)
$$

where $\delta$ denotes the Dirac delta function. As is well known, $H$ can be given a rigorous meaning via quadratic form techniques, and then amounts to an unusual self-adjoint extension of the Laplacean restricted to $C_{0}^{\infty}$-functions whose support does not meet the hyperplanes $q_{j}=q_{k}$.

We shall first discuss the features of $H$ as an operator on $L^{2}\left(\mathbb{R}^{N}\right)$ (the symmetric $L^{2}$-functions). For $\lambda>0$ the interaction is repulsive and one obtains yet another quantum pure soliton system without a classical version. The analysis involved in proving this can be found in the $\mathrm{Ph} . \mathrm{D}$. thesis of Oxford [76]. In fact, he handles the far more complicated attractive case $\lambda<0$. The complications are due to the presence of $M$-body bound states for any $M \leqslant N$, whose center-of-mass wave function reads

$$
\psi\left(q_{1}, \ldots, q_{M}\right)=\prod_{1<i<j<M} \exp \left(\lambda\left|q_{i}-q_{j}\right| / 2\right)
$$

We are now prepared to detail the relation to the $\mathrm{II}_{\mathrm{nr}}$ particle systems. To this end we begin by considering the $N=2$ center-of-mass situation. The key observation is, that the kernel (3.35) can be continued to $g \in(-1 / 2,1 / 2)$; It then corresponds to a self-adjoint extension of the restriction of (3.34) to $C_{0}^{\infty}((0, \infty))$ that differs from the (Friedrichs) extension associated with the choice $g \in(1 / 2,3 / 2)$. For $g \in(-1 / 2,0)$ the operator $\varepsilon$ defined by (3.31) with $E(q, \theta) \rightarrow E(v, g ; \theta, q)$ (cf. (3.35)) does not extend to an isometry onto $\mathcal{T}$. This is due to the presence of a bound state orthogonal to its range, viz.,

$$
\psi(q)=(\operatorname{sh} v q)^{g}, \quad q>0
$$

Setting

$$
g \equiv \lambda / \mu, \quad \mu=2 v
$$

and taking $\mu \rightarrow \infty$, the kernel $E(\mu / 2, \lambda / \mu ; \theta, q)$ converges to

$$
E(q, \theta)=(2 \pi)^{-1 / 2}\left[\left[\frac{2 \theta-i \lambda}{2 \theta+i \lambda}\right]^{1 / 2} \exp (i q \theta)+c . c .\right], \quad q, \theta>0
$$

and the bound state (4.35) to

$$
\psi(q)=\exp (\lambda q / 2), \quad q>0
$$

These are precisely the NLS transform and bound state, transformed from $L^{2}(\mathbb{R})_{s}$ to $L^{2}([0, \infty))$ in the obvious way. Notice that the (formally) attractive/repulsive $\mathrm{II}_{\mathrm{nr}}$ potentials lead to repulsive/attractive NLS 'potentials'!

More generally, we expect (strong) convergence of the $\mathrm{II}_{\mathrm{nr}}$ arbitrary $N$ transforms to the NLS boson transform in the same scaling limit. Apart from the rule of thumb that in soliton theory the two-body situation extends to arbitrary particle number, there are two more solid hints that this should be true. First, the parameter $\mu$ sets the length scale: When it goes to $\infty$, the $\mathrm{II}_{\mathrm{nr}}$ potentials converge to 0 inside the Weyl chamber $G$, cf. (2.18)-(2.23). Hence, one expects that the asymptotics following from (3.21) and (3.44), viz.,

$$
\begin{aligned}
& E(q, \theta) \sim(2 \pi)^{-N / 2} \sum_{\sigma \in S_{N}}(-)^{\sigma} \prod_{\substack{i<j \\
\sigma^{-1}(i)<\sigma^{-1}(j)}} u\left(\frac{1}{2}\left(\theta_{i}-\theta_{j}\right)\right)^{1 / 2} \prod_{\substack{i<j \\
\sigma^{-1}(i)>\sigma^{-1}(j)}} u\left(\frac{1}{2}\left(\theta_{i}-\theta_{j}\right)\right)^{-1 / 2} \\
& \cdot \exp \left(i q \cdot \theta_{\sigma}\right), \quad q_{N} \ll<\cdots<<q_{1}
\end{aligned}
$$

extends to all of $G$ in the limit. Accepting this and noting

$$
-\lim _{\mu \rightarrow \infty} u\left(\mathrm{II}_{\mathrm{nr}}, g=\lambda / \mu ; \theta\right)=\frac{2 \theta-i \lambda}{2 \theta+i \lambda}=u(\mathrm{NLS}, \lambda ; \theta)
$$


(cf. (3.45)), one obtains the NLS transform corresponding to the channel without bound states (up to an overall phase). Indeed, the latter is given by the rhs of (4.39) with $u \rightarrow u$ (NLS) and the factor (-) omitted. (The natural comparison operator for the NLS boson case considered here is the generalized cosine transform.) The second indication of convergence for general $N$ is the bound state behavior for $\mu \rightarrow \infty$ : For $g<0$ and $|g|$ sufficiently small, the $\mathrm{II}_{\mathrm{ar}}$ dynamics has an $M$-body bound state

$$
\psi\left(q_{1}, \ldots, q_{M}\right)=\prod_{1<i<j<M} \operatorname{shr}_{2} \frac{1}{2} \mu\left(q_{i}-q_{j}\right)^{g}, \quad q_{M}<\cdots<q_{1}
$$

which converges to the NLS bound state (4.34) in the above scaling limit.

On account of arguments similar to the ones just presented, we expect that the NLS boson transforms can also be obtained as scaling limits of the $g=2 \mathrm{II}_{\text {rel }}$ transforms. Indeed, from (3.49) we have

$$
-\lim _{\beta \rightarrow 0} u\left(\Pi_{\mathrm{red}}, g=2, \alpha=\pi-\beta \lambda / 2 ; \theta\right)=u(\mathrm{NLS}, \lambda ; \theta)
$$

Recalling $\alpha=\beta v=\beta \mu / 2$, we see that $\mu \rightarrow \infty$ when $\beta \rightarrow 0$, so that this limit is analogous to (4.40). In fact, it is straightforward to verify that for $q, \theta>0$ the $N=2$ kernel $E_{1}(q, \theta)$ (given by (3.38)-(3.42)) converges to the NLS kernel (4.37) in the limit just detailed.

Provided one restricts attention to the $N=2$ case, one can also reach the NLS transform (4.37) via a $g=2 \tilde{I}_{\text {rel }}$ transform. Moreover, this can be done in two essentially different ways. The first scaling limit is the one specified in (4.42), whereas the second one consists in taking $\alpha$ to $\pi / 2, \mu$ to 0 and (hence) $\beta$ to $\infty$; Specifically, one should set $\alpha=(\pi-v \lambda) / 2$. Also, in the second scaling limit one gets convergence to (4.37) with $q$ and $\theta$ interchanged. It is not clear to us whether similar relations are going to persist in these two limits when $N$ is greater than 2 . At any rate, since solitons and antisolitons are distinguishable, one would need some very special linear combinations of the eigenfunctions (which, it should be recalled, have not yet been found for $N>2$ ).

At this point we should mention that the eigenfunctions of the NLS Hamiltonian (4.33) for distinguishable particles are also known [77-79]. (We are not aware of a proof of the Plancherel formula for this case.) However, for $g=2$ the $\bar{s}$ reflection coefficient $r$ vanishes, in contrast to $r$ (NLS). Therefore, it is not likely that the $g=2 \tilde{\mathrm{I}}_{\text {rel }}$ eigenfunctions can lead to the non-symmetrized eigenfunctions of (4.33). On the other hand, it follows from (3.61), (3.62) that one does obtain the NLS transmission and reflection in the limit $(4.40)$. Thus, one may expect to get the complete $L^{2}(\mathbb{R})$ transform via the $N=2 \tilde{\mathrm{I}}_{\mathrm{nr}}$ eigenfunctions. (We have not checked this.)

Let us add one more remark concerning the situation for distinguishable particles. As already mentioned, we have trouble seeing how the $L^{2}\left(\mathbb{R}^{N}\right)$ transform might be reached via type II systems. However, it may well be that the desired transform can be obtained via a linear combination of $\mathrm{II}_{\mathrm{nr}}$ eigenfunctions, as a generalization of the picture for the NLS boson transforms already sketched. (The latter picture, to be sure, is very plausible but not proven.) Again, for $N=2$ this can be achieved. Indeed, the kernel

$$
E(q, \theta) \equiv E(\mu / 2, \lambda / \mu ;|\theta|,|q|)+\epsilon(q) \varepsilon(\theta) E(\mu / 2,1-\lambda / \mu ;|\theta|,|q|), \quad q, \theta \in \mathbb{R}^{*}
$$

(recall (3.35)) converges to the even extension of the kernel (4.37) on $L^{2}(\mathbb{R})$ s and to the kernel $(2 / \pi)^{1 /} \sin q \theta$ on $L^{2}(\mathbf{R})_{a}$ for $\mu \rightarrow \infty$; This is the desired result, since (4.33) amounts to the Laplacean on the fermion subspace.

The structure (4.39) of the asymptotics of the $\mathrm{II}_{\mathrm{nr}}$ eigenfunctions goes back to Harish-Chandra's monumental work on harmonic analysis, cf. [31]. As we have recalled above, the NLS boson transform has this structure for all of the wedge G. In the physics literature this form of the eigenfunctions is referred to as the Bethe Ansatz, after Bethe [80], who was the first to obtain such eigenfunctions for the $X X X$ model (isotropic Heisenberg chain). From a mathematical viewpoint this model (more precisely, its infinite-volume ground-state representation) is very similar to the attractive NLS boson model. In fact, Oxford's solution to the Plancherel problem mentioned above [76] was patterned after previous work on the $X X X$ model by Babbitt and Thomas, who in an impressive series of 
papers [81-84] not only proved the Plancherel formula, but also obtained mathematically rigorous results concerning soliton scattering and conserved quantities. Presently, work by Babbitt and Gutkin is under way [85] which promises to elevate the $X X Z$ model to a comparable mathematical level.

It so happens that the $X X Z$ two-magnon $S$-matrix can be transformed to a difference kernel (cf. e.g. Ch. 1 in Gaudin's monograph [86]). It then amounts to the $g=2 \mathrm{II}_{\text {rel }}$ two-soliton $S$-matrix (3.49) for anisotropy parameter $\Delta \in(0,1)$ and to the $g=2 \hat{I I I}_{\text {rel }}$ two-soliton $S$-matrix [44] for $\Delta \in(1, \infty)$. This may be compared to the equality of the NLS two-soliton $S$-matrix (4.40) and the $g=2 \mathrm{II}_{\mathrm{nr}} S$-matrix (3.45). This is only one reason why we believe that there might be a way to tie in scaling limits of 'relativistic' eigenfunctions with the Bethe transforms of the $X X Z$ model, as a generalization of the links between degenerate $\mathrm{II}_{\mathrm{nr}}$ particle systems and the NLS Bethe transforms sketched above. Since one is dealing with lattice models, one would probably need limits of systems of type $\mathrm{III}_{\text {rel }}$ or $\mathrm{IV}_{\text {rel }}$. However, to date we have not been able to find a clearcut connection even for $N=2$.

By now, those readers still with us may well be tired of our hunches. Instead of indulging in further speculations, let us finish by pointing out some connections that do have solid proofs (though one may question the assumptions on which these are based). First, the $S$-matrix (3.56) with $\tau=\pi / 2$ is just the soliton-soliton $S$-matrix of the quantum sine-Gordon model [45]. As pointed out by Zamolodchikov [87], this $S$-matrix is very closely related to the 6-vertex model free energy (cf. e.g. p. 148 in Baxter's monograph [88]). Second, when one proceeds as sketched in the paragraph containing (3.54), but now for the systems dual to the $\mathrm{IV}_{\text {rel }}$ systems, one obtains [44] an $S$-matrix that has a specialization related in a similar way to the 8-vertex model free energy $[87,88]$. Third, for $\tau=\pi / 2$ the boundstate spectrum (3.71) amounts to the sine-Gordon soliton-antisoliton bound state spectrum [45].

We do not know how to compare the $\tau=\pi / 2$ soliton-soliton wave functions (3.38)-(3.42) or the lowest-energy soliton-antisoliton bound state (3.72) to any previous results in the physics literature. Indeed, physicists appear to be convinced that a relativistic quantum mechanics description of the sine-Gordon/massive Thirring model is impossible.

\section{ACKNOWLEDGEMENTS}

We would like to thank R. Goodman, G. Heckman, E. Thomas, L. Woronowicz and, especially, T. Koornwinder for illuminating discussions on harmonic analysis, special functions, and quantum groups. We also take this opportunity to thank S. Albeverio and A. Hurst, who responded to a Query in Notices of the AMS, making us aware of Nörlund's monograph [38] and the references [39-41].

\section{REFERENCES}

1. S.N.M. RuUJSEnAARS, H. SChNeIDER, A new class of integrable systems and its relation to solitons, Ann. Phys. (NY) 170, 370-405 (1986).

2. S.N.M. RUIUSENAARS, Complete integrability of relativistic Calogero-Moser systems and elliptic function identities, Commun. Math. Phys. 110, 191-213 (1987).

3. S.N.M. RuUSENAARS, Relativistic Toda systems, to appear.

4. M.A. Olshanetsky, A.M. Perelomov, Classical integrable finite-dimensional systems related to Lie algebras, Phys. Reps. 71, 313-400 (1981).

5. M.A. Olshanetsky, A.M. Perelomov, Quantum integrable systems related to Lie algebras, Phys. Reps. 94, 313-400 (1983).

6. S.N.M. RUUUSENAARS, Action-angle maps and scattering theory for some finite-dimensional integrable systems, The pure soliton case, Commun. Math. Phys. 115, 127-165 (1988).

7. S.N.M. RUUSENAARS, Action-angle maps and scattering theory for some finite-dimensional integrable systems. II Solitons, antisolitons, and their bound states, to appear.

8. S.N.M. RUUUSENAARS, Action-angle maps and scattering theory for some finite-dimensional integrable systems. III Sutherland type systems and their duals, to appear.

9. V.I. ARNOLD, Mathematical methods of classical mechanics, Springer, Berlin (1978).

10. M. REED, B. SIMON, Methods of modern mathematical physics. III Scattering theory, Academic Press, New York, (1979). 
11. W. ThmRrnng, Lehrbuch der mathematischen Physik. I. Klassische dynamische Systeme, Springer, Berlin (1977).

12. S.N.M. RUUUSENAarS, On one-dimensional integrable quantum systems with infinitely many degrees of freedom, Ann. Phys. (NY) 128, 335-362 (1980).

13. S.N.M. RUUSENAARS, A positive energy dynamics and scattering theory for directly interacting relativistic particles, Ann. Phys. (NY) 126, 399-449 (1980).

14. J. MOSER, Three integrable Hamiltonian systems connected with isospectral deformations, Adv. in Math. 16, 197-220 (1975).

15. J. MOSER, Finitely many mass points on the line under the influence of an exponential potentialan integrable system, pp. 467-497 in: Dynamical systems, theory and applications (J. Moser ed.), Lect. Notes in Physics Vol. 38, Springer, Berlin (1975).

16. H. Arrault, H.P. McKran, J. Moser, Rational and elliptic solutions of the Korteweg-de Vries equation and a related many-body problem, Commun. Pure Appl. Math. 30, 95-148 (1977).

17. D. KAZHDAN, B. KOSTANT, S. STRRNBERG, Hamiltonian group actions and dynamical systems of Calogero type, Commun. Pure Appl. Math. 31, 481-507 (1978).

18. M. ADLRR, Completely integrable systems and symplectic actions, J. Math. Phys. 20, 60-67 (1979).

19. P.P. KuUss, Factorization of the classical and the quantum $S$ matrix and conservation laws, Theor. Math. Phys. 26, 132-137 (1976).

20. F. CAlogero, Exactly solvable one-dimensional many-body problems, Lett. Nuovo Cim. 13, $411-416(1975)$.

21. I.M. KrICHEVER, Elliptic solutions of the Kadomtsev-Petviashvili equation and integrable systems of particles, Func. Anal. Appl. 14, 282-290 (1980).

22. M. Bruschn, F. CALOGERo, The Lax representation for an integrable class of relativistic dynamical systems, Commun. Math. Phys. 109, 481-492 (1987).

23. H. FuAsCrKa, The Toda lattice. II Existence of integrals, Phys. Rev. B9, 1924-1925 (1974).

24. M. ADLER, P. VAN MOERBEKR, Linearization of Hamiltonian systems, Jacobi varieties, and representation theory, Adv. in Math. 38, 318-379 (1980).

25. M. TODA, Theory of nonlinear lattices, Springer, Berlin (1981).

26. M. BRuSCHI, O. RAGNISCO, Lax representation and complete integrability for the periodic relativistic Toda lattice, Phys. Lett. A134, 365-370 (1989).

27. V.I. Inozemrsev, The finite Toda lattices, Commun. Math. Phys. 121, 629-638 (1989).

28. E.T. Whitraker, G.N. Watson, A course of modern analysis, Cambridge University Press, Cambridge, (1973).

29. O. RAGNISCO, private communication.

30. F.A. BEREZIN, Laplace operators on semisimple Lie groups, Amer. Math. Soc. Transl. 21, 239-339 (1962).

31. S. Helgason, Groups and geometric analysis, Academic Press, New York, (1984).

32. H. SCHLICHTRRULL, Hyperfunctions and harmonic analysis on symmetric spaces, Birkhäuser, Boston (1984).

33. A. TERRAS, Harmonic analysis on symmetric spaces and applications II, Springer, Berlin (1988).

34. G.J. HeCKMan, E.M. Opdam, Root systems and hypergeometric functions I, Comp. Math. 64, 329-352 (1987).

35. G.J. HeCKMAN, Root systems and hypergeometric functions II, Comp. Math. 64, 353-373 (1987).

36. E.M. OPDAM, Root systems and hypergeometric functions III, Comp. Math. 67, $21-49$ (1988).

37. E.M. OPDAM, Root systems and hypergeometric functions IV, Comp. Math. 67, 191-209 (1988).

38. N.E. NöRuUND, Vorlesungen über Differenzenrechnung, Springer, Berlin (1924).

39. R. Jost, Lineare Differenzengleichungen mit periodischen Koeffizienten, Comm. Math. Helv. 28, 173-185 (1954).

40. S. ALBeverio, Analytische Lösung eines idealisierten Stripping- oder Beugungsproblems, Helv. Phys. Acta 40, 135-184 (1967). 
41. J.B. MC GUIRE, C.A. HuRST, The scattering of three impenetrable particles in one dimension, J. Math. Phys. 13, 1595-1607 (1972).

42. G.K. IMMINK, Asymptotics of analytic difference equations, Lect. Notes in Math. Vol. 1085, Springer, Berlin (1984).

43. S.N.M. RUUUSENAARS, to appear.

44. S.N.M. RUIJSENAARS, First-order analytic difference equations and integrable quantum systems, to appear.

45. A.B. Zamolodchixov, A.B. Zamolodchrixov, Factorized $S$-matrices in two dimensions as the exact solutions of certain relativistic quantum field theory models, Ann. Phys. (NY) 120, 253-291 (1979).

46. R. ASKEY, M.E.-H. ISMAII, A generalization of ultraspherical polynomials, pp. 55-79 in: Studies in Pure Math. (P. Erdös, ed.), Birkhäuser, Boston (1983).

47. R. ASKEY, J. WIISON, Some basic hypergeometric orthogonal polynomials that generalize Jacobi polynomials, Memoirs Amer. Math. Soc., Vol. 54, Providence (1985).

48. T. KOORNWINDER, private communication.

49. R. ASKEY, J. WILSON, A set of orthogonal polynomials that generalize the Racah coefficients or 6-j symbols, SIAM J. Math. Anal. 10, 1008-1016 (1979).

50. I.G. MCDONALD, Orthogonal polynomials associated to root systems, Oxford preprint (1988).

51. S.L. WoronowiCZ, Twisted SU(2) group. An example of a non-commutative differential calculus, Publ. RIMS 23, 117-181 (1987).

52. S.L. Woronowicz, Compact matrix pseudogroups, Commun. Math. Phys. 111, 613-665 (1987).

53. S.L. Woronowicz, Tannaka-Krein duality for compact matrix pseudogroups. Twisted SU(N) groups, Invent. Math. 93, 35-76 (1988).

54. T. KOORNWINDER, Continuous $q$-Legendre polynomials are spherical matrix elements of irreducible representations of the quantum SU(2) group, CWI Quarterly 2, 171-173 (1989).

55. S.N.M. RUUUSENAARS, Generalized Lamé functions, to appear.

56. R. GOODMAN, N.R. WALLACH, Classical and quantum mechanical systems of Toda-lattice type III. Joint eigenfunctions of the quantized systems, Commun. Math. Phys. 105, 473-509 (1986).

57. R.K. Bullough, S. Olafsson, Complete integrability of the integrable models: quick review, pp. 329-334 in: Proceedings of the 1988 Swansea IAMP Conference (B. Simon, A. Truman, I.M. Davies, eds.), IOP Publishing, Bristol (1989).

58. S.N.M. RuUSENAARS, Relativistic Calogero-Moser systems and solitons, pp. 182-190 in: Topics in soliton theory and exactly solvable nonlinear equations (M. Ablowitz, B. Fuchssteiner, M. Kruskal, eds.), World Scientific, Singapore (1987).

59. V.E. Zakharov, L.D. FADDeEv, Korteweg-de Vries equation, a completely integrable Hamiltonian system, Func. Anal. Appl. 5, 280-287 (1972).

60. H.F. FLASCHKA, A.C. NEWELl, Integrable systems of nonlinear evolution equations, pp. 355-440 in: Dynamical systems, theory and applications (J. Moser, ed.), Lect. Notes in Physics Vol. 38, Springer, Berlin (1975).

61. H.F. Flaschra, D.W. McLaughin, Some comments on Băcklund transformations, canonical transformations, and the inverse scattering method, pp. 253-295 in: Bäcklund transformations ( $\mathbb{R}$. Miura, ed.), Lect. Notes in Math. Vol. 515, Springer, Berlin (1976).

62. L.D. FADDEEV, L.A. TAKrmajan, Hamiltonian methods in the theory of solitons, Springer, Berlin (1987).

63. V.S. Buslaev, L.D. Faddeev, L.A. Takrtajan, Scattering theory for the Korteweg-de Vries (KdV) equation and its Hamiltonian interpretation, Physica 18D, 255-266 (1986).

64. F. MAGRI, A simple model of the integrable Hamiltonian equation, J. Math. Phys. 19, 1156-1162 (1978).

65. B.A. KUPERSHMmT, G. WIIsON, Modifying Lax equations and the second Hamiltonian structure, Invent. Math. 62, 403-436 (1981).

66. C.S. GARDNER, Korteweg-de Vries equation and generalizations. IV. The Korteweg-de Vries 
equation as a Hamiltonian system, J. Math. Phys. 12, 1548-1551 (1971).

67. L.D. Faddere, L.A. Takrtajan, Poisson structure for the KdV equation, Lett. Math. Phys. 10, 183-188 (1985).

68. W. ECKHAus, P. SchuUR, The emergence of solitons of the Korteweg-de Vries equation from arbitrary initial conditions, Math. Meth. Appl. Sci. 5, 97-116 (1983).

69. E. DaTE, M. JMBBo, M. KashiwarA, T. MrWA, Landau-Lifshitz equation: solitons, quasi-periodic solutions and infinite-dimensional Lie algebras, J. Phys. A16, 221-236 (1983).

70. S.N.M. RUUUSENAARS, Scattering theory for the Federbush, massless Thirring and continuum Ising models, J. Func. Anal. 48, 135-171 (1982)

71. S.N.M. RuUSENAARS, The Wightman axioms for the fermionic Federbush model, Commun. Math. Phys. 87, 181-228 (1982).

72. J. PAIMgR, C. TraCY, Two-dimensional Ising correlations: convergence of the scaling limit, Adv. Appl. Math. 2, 329-388 (1981).

73. R. SCHOR, M.O' CARROLI, The scaling limit and Osterwalder-Schrader axioms for the twodimensional Ising model, Commun. Math. Phys. 84, 153-170 (1982).

74. J. PALMER, C. TRACY, Two-dimensional Ising correlations: the SMJ analysis, Adv. Appl. Math. 4, 46-102 (1983).

75. S.N.M. RUUISENAARS, Integrable quantum field theories and Bogoliubov transformations, Ann. Phys. (NY) 132, 328-382 (1981).

76. S. OXFORD, The Hamiltonian of the quantized nonlinear Schrödinger equation, $\mathrm{Ph} . \mathrm{D}$. thesis, Los Angeles (1979).

77. J.B. MCGurRe, Study of exactly soluble one-dimensional $N$-body problems, J. Math. Phys. 5 , 622-636 (1964).

78. C.N. YANG, Some exact results for the many-body problem in one dimension with repulsive delta-function interaction, Phys. Rev. Lett. 19, 1312-1315 (1967).

79. C.N. YANG, $S$ matrix for the one-dimensional $N$-body problem with repulsive of attractive $\delta$ function interaction, Phys. Rev. 168, 1920-1923 (1968).

80. H.A. BETHE, Zur Theorie der Metalle. I. Eigenwerte und Eigenfunktionen der linearen Atomkette, Zeitschr. f. Physik, 71, 205-226 (1931).

81. L.E. THOMAS, Ground state representation of the infinite one-dimensional Heisenberg ferromagnet I, J. Math. Anal. Appl. 59, 392-414 (1977).

82. D. BABbIT, L. ThOMAS, Ground state representation of the infinite one-dimensional Heisenberg ferromagnet II. An explicit Plancherel formula, Commun. Math. Phys. 54, 255-278 (1977).

83. D. BABBIT, L. Thomas, Ground state representation of the infinite one-dimensional Heisenberg ferromagnet III. Scattering theory, J. Math. Phys. 19, 1699-1704 (1978).

84. D. BABBIT, L. Thomas, Ground state representation of the infinite one-dimensional Heisenberg ferromagnet IV. A completely integrable system, J. Math. Anal. Appl. 72, 305-328 (1979).

85. D. BABbIrT, E. GuTKIN, The Plancherel formula for the infinite $X X Z$ Heisenberg spin chain, Los Angeles preprint (1989).

86. M. Gaudin, La fonction d'onde de Bethe, Masson, Paris (1983).

87. A.B. ZAMOLODCHIKov, $Z_{4}$-symmetric factorized $S$-matrix in two space-time dimensions, Commun. Math. Phys. 69, 165-178 (1979).

88. R.J. BAXTER, Exactly solved models in statistical mechanics, Academic Press, New York (1982). 